\title{
CARMA OBSERVATIONS OF PROTOSTELLAR OUTFLOWS IN NGC 1333
}

\author{
Adele L. Plunkett ${ }^{1,6}$, Héctor G. Arce ${ }^{1}$, Stuartt A. Corder ${ }^{2}$, Diego Mardones ${ }^{3}$, \\ AnNeIla I. SARgENT ${ }^{4}$, AND SCOTt L. SChNeE 5 \\ ${ }^{1}$ Department of Astronomy, Yale University, P.O. Box 208101, New Haven CT 06520, USA; adele.plunkett@yale.edu \\ 2 Joint ALMA Observatory, Av. Alonso de Córdova 3107, Vitacura, Santiago, Chile \\ ${ }^{3}$ Departameto de Astronomía, Universidad de Chile, Casilla 36-D, Santiago, Chile \\ ${ }^{4}$ Astronomy Department, California Institute of Technology, 1200 East California Boulevard, Pasadena, CA 91125, USA \\ 5 National Radio Astronomy Observatory, 520 Edgemont Road, Charlottesville, VA 22903, USA \\ Received 2013 February 25; accepted 2013 July 8; published 2013 August 12
}

\begin{abstract}
We present observations of outflows in the star-forming region NGC 1333 using the Combined Array for Research in Millimeter-Wave Astronomy (CARMA). We combined the ${ }^{12} \mathrm{CO}$ and ${ }^{13} \mathrm{CO}(1-0)$ CARMA mosaics with data from the $14 \mathrm{~m}$ Five College Radio Astronomy Observatory to probe the central, most dense, and active region of this protostellar cluster at scales from $5^{\prime \prime}$ to $7^{\prime}$ (or $1000 \mathrm{AU}$ to $0.5 \mathrm{pc}$ at a distance of $235 \mathrm{pc}$ ). We map and identify ${ }^{12} \mathrm{CO}$ outflows, and along with ${ }^{13} \mathrm{CO}$ data we estimate their mass, momentum, and energy. Within the $7^{\prime} \times 7^{\prime}$ map, the $5^{\prime \prime}$ resolution allows for a detailed study of morphology and kinematics of outflows and outflow candidates, some of which were previously confused with other outflow emission in the region. In total, we identify 22 outflow lobes, as well as 9 dense circumstellar envelopes marked by continuum emission, of which 6 drive outflows. We calculate a total outflow mass, momentum, and energy within the mapped region of $6 M_{\odot}, 19 M_{\odot} \mathrm{km} \mathrm{s}^{-1}$, and $7 \times 10^{44} \mathrm{erg}$, respectively. Within this same region, we compare outflow kinematics with turbulence and gravitational energy, and we suggest that outflows are likely important agents for the maintenance of turbulence in this region. In the earliest stages of star formation, outflows do not yet contribute enough energy to totally disrupt the clustered region where most star formation is happening, but have the potential to do so as the protostellar sources evolve. Our results can be used to constrain outflow properties, such as outflow strength, in numerical simulations of outflow-driven turbulence in clusters.
\end{abstract}

Key words: ISM: individual objects (NGC 1333) - ISM: jets and outflows - ISM: molecules - stars: formation - stars: protostars - techniques: interferometric

Online-only material: color figures

\section{INTRODUCTION}

Outflows are generally understood as a necessary component of the star formation process (e.g., Shu et al. 1987). They expel mass, remove the excess angular momentum accumulated during gravitational infall, and allow accretion onto a forming protostar. Not only does an outflow play an important role in the formation of the protostar which drives it, an outflow also likely impacts its surrounding environment, and in dense cluster environments where many protostars are driving outflows, the fate of the cluster may depend on the level of outflow activity. For example, energetic outflows inject momentum and energy into the cloud, and in the process they may disperse the surrounding gas and feed turbulent motions (Arce et al. 2007). Given that the majority of stars form within embedded clusters with many members (Lada \& Lada 2003), understanding these environments is vital to understanding the formation of most stars.

Particularly in low-mass star-forming regions, outflows may disrupt the surrounding environment enough to limit the lifetime of their parent molecular cloud (Hartmann et al. 2001). Recent analytical and numerical studies have shown that outflows can interact with the cloud and drive turbulent motions very efficiently (Matzner 2007; Nakamura \& Li 2007; Cunningham et al. 2009; Carroll et al. 2009). Other numerical studies suggest that outflows disrupt dense clumps and affect cloud structure, but are inefficient at driving turbulence within a cloud (Banerjee et al. 2007). The question remains as to whether outflows

\footnotetext{
6 NSF Graduate Research Fellow.
}

have enough energy and momentum to alter the surrounding molecular gas and trigger the formation of stars (as indicated by the models of Foster \& Boss 1996), or possibly disperse the cluster gas (e.g., Benedettini et al. 2004; Arce et al. 2010). While the underlying physics of outflow-cloud interactions can be simulated, observations sensitive to a range of spatial scales to probe distinct outflows and the surrounding cloud are critical for constraining characteristics of protostellar clusters, and specifically numerical simulations that model outflow-induced turbulence.

Here we present observations of $\mathrm{CO}$ in the low-mass starforming region NGC 1333 with the primary goal to identify and characterize outflow activity. We also present continuum observations made simultaneously with the $\mathrm{CO}$ observations, and although the continuum data were not the priority of our observing plan, they allow a qualitative and quantitative study of several outflow-driving sources in the region. In total, we identify 22 outflow lobes, as well as 9 dense circumstellar envelopes marked by continuum emission within a $\sim 0.23 \mathrm{pc}^{2}$ area. Our observations probe physical scales over two orders of magnitude and outflow velocities up to $\pm 10 \mathrm{~km} \mathrm{~s}^{-1}$ from the cloud velocity, which we find necessary to study the distinct morphologies and kinematics of previously known outflows as well as new outflow candidates that until now were confused with other outflow emission in the region. We measure mass, momentum, and energy of individual outflows, as well as mean characteristics of outflows in our map, providing important parameters for numerical simulations of clustered star formation. We also compare outflow energetics with turbulence 


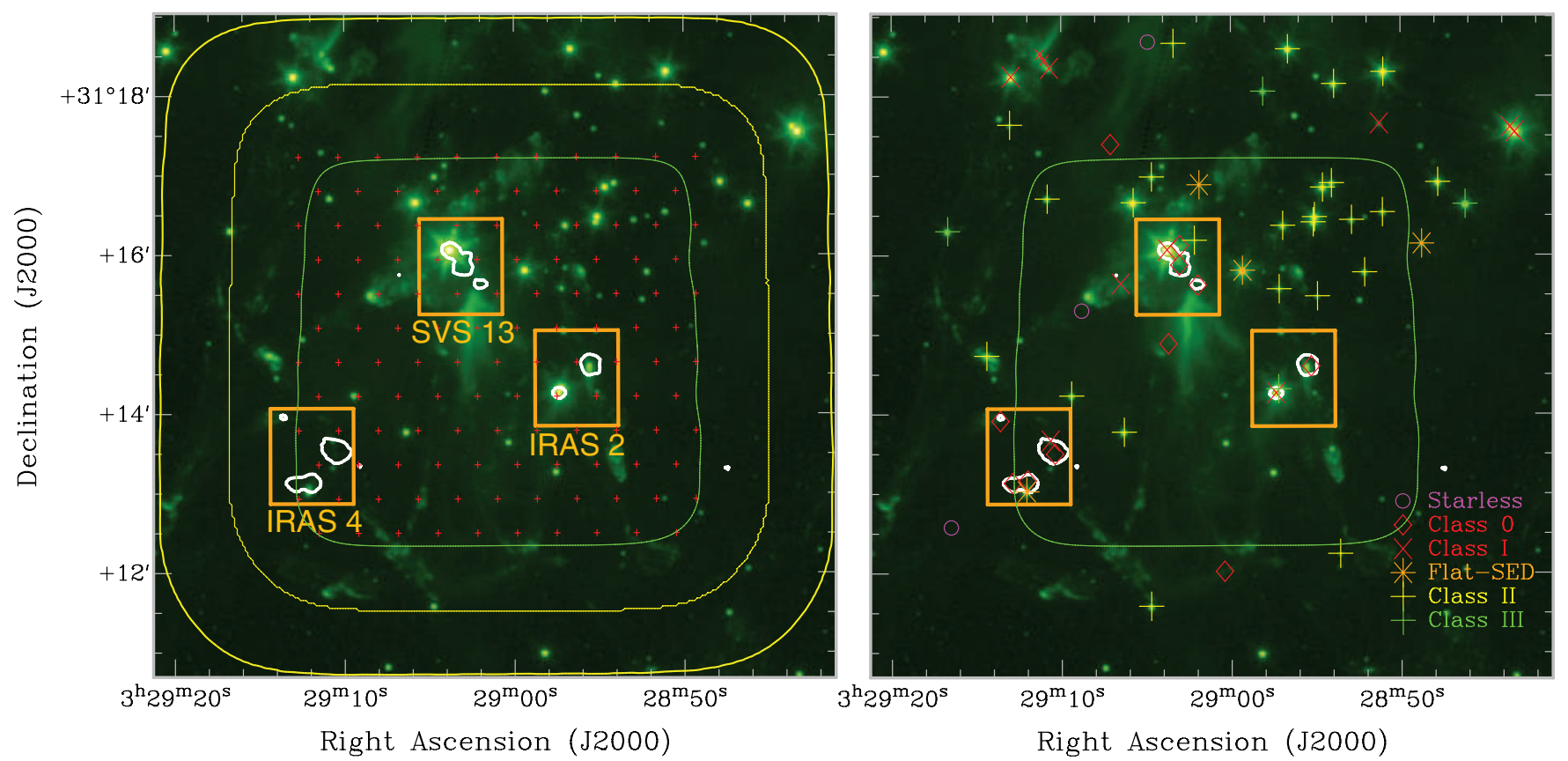

Figure 1. Spitzer/IRAC $4.5 \mu \mathrm{m}$ image of NGC 1333 (Gutermuth et al. 2008), showing the region we mapped. More details about our observations are given in Table 1. Left: Red crosses show the 126 mosaic pointings in a hexagonal-packed pattern that comprise our CARMA maps (see Section 2.1). Green contours indicate the region with constant sensitivity in our CARMA mosaic (see Table 1 for rms values), while sensitivity is degraded at the edges beyond the mosaic pattern. The yellow (outermost) contours mark the outer edge of the mapped region, where sensitivity (between the yellow contours) is $12 \mathrm{mJy} \mathrm{beam}^{-1}, 1.1 \mathrm{Jy} \mathrm{beam}^{-1}$, and $0.7 \mathrm{Jy}$ beam ${ }^{-1}$ for continuum, ${ }^{12} \mathrm{CO}$, and ${ }^{13} \mathrm{CO}$, respectively. Orange boxes indicate the regions which are shown in $2.7 \mathrm{~mm}$ continuum in Figure 3 , with white contours marking $4 \sigma$ continuum emission (i.e., the lowest-level contour in Figure 3). Right: YSOs within the region we mapped, identified in Table 2. White contours and orange boxes are the same as in the left panel. The green contour is the same as the inner green contour in the left panel, indicating the region we mapped with greatest sensitivity.

(A color version of this figure is available in the online journal.)

and gravity in the region. We suggest that outflows have the potential to maintain turbulence in this region, and eventually may drive enough energy to contribute toward disruption of the central, clustered region where most star formation is happening. NGC 1333 is the first in a survey of star-forming regions that we plan to study ranging in mass and evolutionary stage, in order to investigate the impact of outflows on their surrounding environments, given environments with a range of characteristics.

\subsection{Description of the Region: NGC 1333}

NGC 1333, a reflection nebula on the near surface of the L1450 dark cloud (Lynds 1962), is considered by many to be the prototypical, nearby cluster-forming region (e.g., Walawender et al. 2008; Padoan et al. 2009, and references therein). It is the most active region of low-mass star formation in the Perseus molecular cloud complex and the nearest largemembership ( $>100$ ) young cluster. A variety of works have contributed to identifying and characterizing the young stellar object (YSO) members of this cluster. Strom et al. (1976) ${ }^{7}$ presented NGC 1333 as part of a survey mapping infrared dark clouds in IR at $2.2 \mu \mathrm{m}$ ( $K$ band), in addition to photometry at $J, H$, and $L$ bands for identified sources within the cloud. Later Jennings et al. (1987) made IRAS observations at 50 and $100 \mu \mathrm{m}$ and revealed nine distinct sources in NGC 1333, five of which are within our mapped region. Based on near-IR photometry, Aspin et al. (1994) suggested that the pre-mainsequence (PMS) sources in NGC 1333 are clustered around

\footnotetext{
7 Throughout we give specific source labels from Strom et al. (1976) as SVS, rather than SSV as was the convention followed by some other works following Herbig \& Jones (1983).
}

SVS 13, where many studies of clustered star formation have since targeted their efforts. Infrared excess is found in $61 \%$ of PMS sources, and Lada et al. (1996) conclude that the cluster has an age of $1-2 \times 10^{6} \mathrm{yr}$ or less. Further, given the current stellar mass $\left(45 M_{\odot}\right)$ in NGC 1333, they suggest that if star formation continues at the current rate $\left(4.5 \times 10^{-5} M_{\odot} \mathrm{yr}^{-1}\right)$ for the next five million years, NGC 1333 could develop into a very rich cluster, reminiscent of IC 348.

Counterparts of the IR and near-IR sources throughout NGC 1333 were detected in radio with the Very Large Array (VLA; Snell \& Bally 1986; Rodríguez et al. 1997, 1999); in submillimeter (sub-mm) with SCUBA/JCMT (Sandell \& Knee 2001); and in sub-arcsec mm observations with BIMA (Looney et al. 2000). For a more thorough review of the region, we refer the reader to Walawender et al. (2008).

Within NGC 1333 are a large number of molecular outflows, Herbig-Haro $(\mathrm{HH})$ objects, and $\mathrm{H}_{2}$ knots (e.g., Bally et al. 1996; Sandell \& Knee 2001; Davis et al. 2008). Based on these indicators, we consider this cluster ideal for studying outflow-cloud interactions. To date, molecular outflows in NGC 1333 have either been (1) observed individually with highresolution, or (2) mapped across the extended region with lowerresolution. Here we present for the first time high-resolution observations that also capture the complex web of outflow activity across the central $\sim 7^{\prime} \times 7^{\prime}$ region of NGC 1333 .

Figure 1 shows the Spitzer/IRAC $4.5 \mu \mathrm{m}$ image of NGC 1333 (Gutermuth et al. 2008), making evident the intricate outflow activity particularly in the central region. We focus on the central $\sim 7^{\prime} \times 7^{\prime}\left(\sim 0.23 \mathrm{pc}^{2}\right.$ at $\left.235 \mathrm{pc}\right)$ region of NGC 1333 where 55 YSOs and 3 starless cores are found (see Table 2), at a density of about $250 \mathrm{YSOs} \mathrm{pc}^{-2}$. This region, approximately centered on SVS 13, has one of the highest protostellar densities 
Table 1

CARMA Maps Summary

\begin{tabular}{|c|c|c|c|c|c|c|c|c|c|}
\hline \multirow[t]{2}{*}{ Line } & \multirow{2}{*}{$\begin{array}{c}\text { Rest Frequency } \\
(\mathrm{GHz})\end{array}$} & \multicolumn{2}{|c|}{ HPBW } & \multirow{2}{*}{$\begin{array}{c}\text { Beam PA } \\
\left({ }^{\circ}\right)\end{array}$} & \multirow{2}{*}{$\begin{array}{c}v_{\min } \\
\left(\mathrm{km} \mathrm{s}^{-1}\right)\end{array}$} & \multirow{2}{*}{$\begin{array}{c}v_{\max } \\
\left(\mathrm{km} \mathrm{s}^{-1}\right)\end{array}$} & \multirow{2}{*}{$\begin{array}{c}\text { Channel Width } \\
\qquad\left(\mathrm{km} \mathrm{s}^{-1}\right)\end{array}$} & \multirow{2}{*}{$\begin{array}{c}\text { Bandwidth } \\
(\mathrm{MHz})\end{array}$} & \multirow{2}{*}{$\begin{array}{c}\text { rms } \\
\left(\mathrm{Jy} \mathrm{beam}^{-1}\right)\end{array}$} \\
\hline & & $\operatorname{maj}\left({ }^{\prime \prime}\right)$ & $\min \left({ }^{\prime \prime}\right)$ & & & & & & \\
\hline${ }^{12} \mathrm{CO}$ & 115.27 & 5.7 & 4.7 & 82.7 & -2.3 & 17.6 & 0.32 & 7.69 & 0.2 \\
\hline${ }^{13} \mathrm{CO}$ & 110.20 & 5.9 & 5.0 & 72.9 & -0.9 & 17.6 & 0.33 & 7.69 & 0.1 \\
\hline Continuum & 112.7 & 6.5 & 5.3 & 84.0 & $\ldots$ & $\ldots$ & $\ldots$ & 1531 & 0.002 \\
\hline
\end{tabular}

in the entire NGC 1333 region and overall Perseus cloud, which have average densities of about $34 \mathrm{YSO} \mathrm{pc}^{-2}$ and $6 \mathrm{YSO} \mathrm{pc}^{-2}$, respectively (Jørgensen et al. 2006).

In Figure 1 and Table 2 we show and list the previously identified YSOs within the region we mapped, in an attempt to synthesize the vast literature related to this region and their subsequent naming conventions. In the table we include references which present survey-like observations of multiple sources within the region that we observed, as well as relevant naming conventions which aid the discussion of this paper and cross-references with the previous literature.

Several distances have been reported for this region, ranging from 220 pc (Černis 1990) to 350 pc (Herbig \& Jones 1983). Here we adopt a distance of $235 \pm 18 \mathrm{pc}$, which was determined by Hirota et al. (2008) based on parallax measurements of the $\mathrm{H}_{2} \mathrm{O}$ maser in NGC 1333 SVS 13. This distance is also consistent with the distance of $250 \mathrm{pc}$ assumed in other studies of the region (e.g., Enoch et al. 2006; Evans et al. 2009; Curtis et al. 2010a; Arce et al. 2011). In a Perseus-wide study of sub-mm cores, Hatchell et al. (2005) assumed a distance of 320 pc, based on the Hipparcos distance to clusters within the Perseus OB2 association (de Zeeuw et al. 1999). However, Enoch et al. (2006) cite evidence that NGC 1333 may lie in the foreground of the more distant Perseus OB2 association, or that the Perseus cloud may span a range of distances.

\section{OBSERVATIONS AND DATA}

\subsection{CARMA Observations}

We observed with the Combined Array for Research in Millimeter-Wave Astronomy (CARMA) in the D- and E-array configurations, mapping a 8.'4 $\times 8.2$ region of NGC 1333, centered at R.A. $=03^{\mathrm{h}} 29^{\mathrm{m}} 01^{\mathrm{s}}$, decl. $=+31^{\circ} 15^{\prime} 00^{\prime \prime}$. In total, we observed $41.6 \mathrm{hr}$ with the D-array configuration and $15.7 \mathrm{hr}$ with the E-array configuration. Combining the D- and E-array configuration observations produced a dataset with baselines ranging from 8.5 to $148 \mathrm{~m}$. CARMA is a heterogeneous array that, at the time of observations in 2008 October and 2009 March, consisted of nine antennas with diameters of $6.1 \mathrm{~m}$ and six antenna with diameters of $10.2 \mathrm{~m}$ (with half-power beam widths (HPBWs) of $100^{\prime \prime}$ and $60^{\prime \prime}$ at $115 \mathrm{GHz}$, respectively). Our mosaic consisted of 126 pointings in a hexagonalpacked pattern, with horizontal spacing of $30^{\prime \prime}$ and vertical spacing of 25".8. This provides better than Nyquist sampling considering the $60^{\prime \prime} \mathrm{HPBW}$ of the $10.2 \mathrm{~m}$ antennas. The resulting synthesized beams and the rms of the maps are given in Table 1.

We simultaneously observed the $J=1-0$ transitions of ${ }^{12} \mathrm{CO}(115.27 \mathrm{GHz})$ in the upper sideband (USB), and ${ }^{13} \mathrm{CO}$ $(110.20 \mathrm{GHz})$ and $\mathrm{C}^{18} \mathrm{O}(109.78 \mathrm{GHz})$ in the lower sideband (LSB). The spectral windows of ${ }^{12} \mathrm{CO}$ and ${ }^{13} \mathrm{CO}$ had widths of $8 \mathrm{MHz}$, and the spectral window of $\mathrm{C}^{18} \mathrm{O}$ had width of $2 \mathrm{MHz}$. With 63 channels in each window, the velocity resolutions were
$0.317 \mathrm{~km} \mathrm{~s}^{-1}, 0.332 \mathrm{~km} \mathrm{~s}^{-1}$, and $0.083 \mathrm{~km} \mathrm{~s}^{-1}$ for ${ }^{12} \mathrm{CO},{ }^{13} \mathrm{CO}$, and $\mathrm{C}^{18} \mathrm{O}$, respectively. In Table 1 we summarize the ${ }^{12} \mathrm{CO}$, ${ }^{13} \mathrm{CO}$ and continuum maps, including the spectral setup and the corresponding minimum and maximum velocity of the lines we present here. We note that $\mathrm{C}^{18} \mathrm{O}$ data are not included in the current paper. Our assumed cloud velocity of $v_{\text {cloud }}=8 \mathrm{~km} \mathrm{~s}^{-1}$ (see Section 3.2) corresponds to channels 33 and 28 (of 63 channels total) in the ${ }^{12} \mathrm{CO}$ and ${ }^{13} \mathrm{CO}$ data cubes, respectively. Hence, we detect line wings up to about $\pm 10 \mathrm{~km} \mathrm{~s}^{-1}$ from the cloud velocity, and although the limited velocity range will result in missing the very high velocity emission of a few outflows, we chose the spectral setup that would give us a sufficient combination of both spectral resolution and coverage.

Our correlator setup included one $500 \mathrm{MHz}$ window, with data taken in both the USB and LSB for a total of $1 \mathrm{GHz}$. The central frequencies for the wide-band windows in the LSB and USB observed with the D-array configuration were $109.9 \mathrm{GHz}$ and $115.5 \mathrm{GHz}$, respectively, and the central frequencies for the windows observed with the E-array configuration were $110.6 \mathrm{GHz}$ and $114.9 \mathrm{GHz}$, respectively. The wide-band windows observed with the D- and E-array configurations did not overlap in frequency coverage, resulting in a total wide-band coverage of $2 \mathrm{GHz}$, including LSB and USB windows from the D- and E-array configuration observations. We omitted $11 \mathrm{D}$-array configuration channels which showed line emission. The total continuum bandwidth in D- and E-array configuration observations, including all line-free channels, was $1.53 \mathrm{GHz}$ with mean frequency of $112.7 \mathrm{GHz}$ (corresponding to wavelength of $2.7 \mathrm{~mm}$ ). In this paper, we present the ${ }^{12} \mathrm{CO}$ and continuum maps, and we use ${ }^{13} \mathrm{CO}$ data to estimate outflow masses (see Section 3.4). We will present the ${ }^{13} \mathrm{CO}$ and $\mathrm{C}^{18} \mathrm{O}$ maps in a future paper discussing the structure of the cloud.

Integrations were $30 \mathrm{~s}$ per mosaic position, and 3 minutes on the phase calibrator, with 50 pointings observed in between each phase calibrator observation. The bright nearby quasar 3C84 was used for phase and gain calibrations, and Uranus was the primary flux calibrator. Data were reduced using MIRIAD (Sault et al. 1995). Anomalous amplitudes and phases were flagged, as were any observations with system temperatures higher than $500 \mathrm{~K}$. The average system temperature was $310 \mathrm{~K}$. No shadowed data were used, nor observations with elevation between 85 and $90 \mathrm{deg}$. Flux and gain calibrations were applied, then data from both configurations were Fourier transformed simultaneously. The maps were inverted using a cell size of $2^{\prime \prime}$, an image size of 129 pixels for each pointing and a linear mosaic operation, and then deconvolved with the maximum entropy deconvolution mosmem. The clean images were then restored with a Gaussian beam.

Since our continuum observations were done simultaneously with the line observations, the maps cover the same area and the observing procedure is the same as described above. We CLEANed the continuum data using the mosaic-specific task mossdi in MIRIAD, and then restored the clean images with a 
Gaussian beam. See Table 1 for the resulting synthesized beam and rms.

We also created maps with units of rms by dividing the intensity map by the sensitivity map (from the MIRIAD task mossen), and correcting for the ratio between the theoretical rms calculated by MIRIAD and the measured rms in the clean image. This allows us to identify (line) emission features with signal greater than $3 \sigma$, given that the sensitivity is not constant at the edges of the map since the data were taken with a heterogenous antenna array (see above), and it is particularly useful for identifying significant emission features near the edge of our map. Maps shown in figures throughout this paper are in units of rms rather than absolute intensity.

Our maps have best sensitivity (with an $\mathrm{rms}$ in the ${ }^{12} \mathrm{CO}$ map of $0.2 \mathrm{Jy} \mathrm{beam}^{-1}$ per channel) in the regions properly Nyquist sampled and covered by the full array of antennas, approximately the central $\sim 6^{\prime} \times 6^{\prime}$ region (i.e., the inner contour shown in Figure 1). Only a subset of the antennas are sensitive to the outer $\sim 1^{\prime}$ regions resulting in degraded sensitivity (with an rms in the ${ }^{12} \mathrm{CO}$ map of $1.1 \mathrm{Jy}$ beam $^{-1}$ per channel). Here we report on molecular outflow emission $>3 \sigma$ per channel that encompasses a region $\sim 7^{\prime} \times 7^{\prime}$ (including part of the map with lower sensitivity), corresponding to an area of $\sim 49 \mathrm{arcmin}^{2}$ $\left(\sim 0.23 \mathrm{pc}^{2}\right)$. However, it should be kept in mind that there might be undetected outflow emission in the outer $\sim 1^{\prime}$ of our maps due to the lower sensitivity in this area.

\subsection{Combining Interferometer and Single Dish Line Data}

We complement the CARMA observations with ${ }^{12} \mathrm{CO}$ and ${ }^{13} \mathrm{CO}$ data in Perseus collected as part of the Coordinated Molecular Probe Line Extinction and Thermal Emission (COMPLETE) Survey of Star Forming Regions (Ridge et al. 2006). Observations were made with the $14 \mathrm{~m}$ Five College Radio Astronomy Observatory (FCRAO) telescope in New Salem, Massachusetts. For a more detailed description of these observations and data, see Ridge et al. (2006). The FCRAO beam size at the frequencies of ${ }^{12} \mathrm{CO}$ and ${ }^{13} \mathrm{CO}(1-0)$ is about $46^{\prime \prime}$.

We combine interferometer (CARMA) and single dish (FCRAO) observations in order to recover flux over a range of spatial scales in the region. The interferometer-only map and the combined map of ${ }^{12} \mathrm{CO}$ are shown in Figure 2. The CARMA map has a resolution of $\sim 5^{\prime \prime}$ (or $\sim 1000 \mathrm{AU}$ at a distance of $235 \mathrm{pc}$ ), and it is necessary for discerning small-scale structure, particularly important for distinguishing outflows and associating them with their driving sources. Engargiola \& Plambeck (1999) and Plambeck \& Engargiola (2000) previously presented BIMA observations of ${ }^{12} \mathrm{CO}$ in NGC 1333, indicating that interferometer mosaic mapping is a feasible technique for identifying outflow features within the molecular cloud. Our CARMA observations follow this method, but with more antenna baselines and collecting area we are now able to achieve higher sensitivity and a more thorough analysis. The FCRAO map is used to image the larger spatial scales, and recover the flux that is resolved out by the interferometer, including total power which corresponds to zero spatial frequency. Specifically, this is critical for recovering flux from large-scale outflows, where gas within outflow lobes is typically composed of extended structures. For a by-eye inspection, we still have the benefit of the interferometer-only map in order to identify certain outflow features, with the interferometer acting as a "spatial filter," while the combined interferometer and single
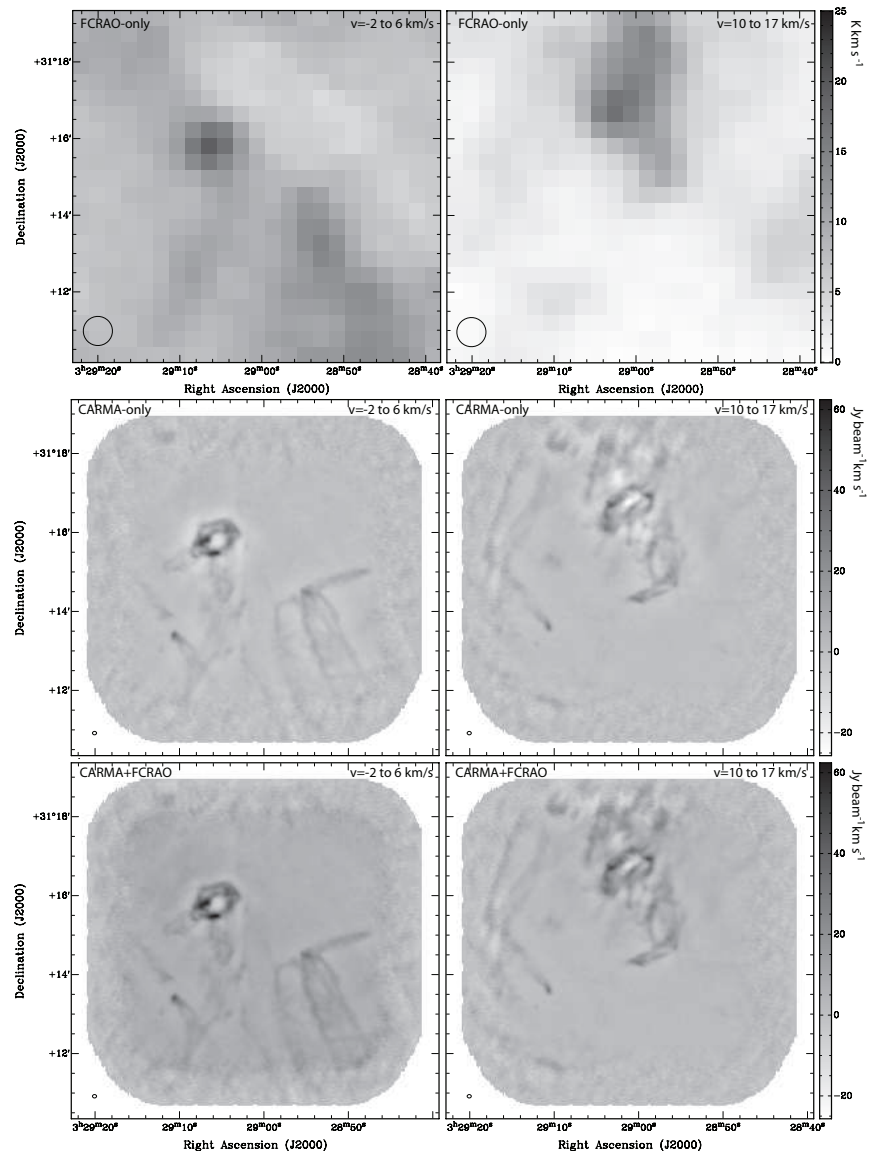

Figure 2. Comparison of same region in FCRAO-only (upper), CARMA-only (middle), and combined CARMA+FCRAO (lower) maps. Left panels show integrated intensity (moment 0 ) maps of blueshifted velocity channels between $V_{\mathrm{LSR}}=-2$ and $6 \mathrm{~km} \mathrm{~s}^{-1}$, and right panels show integrated intensity maps of redshifted velocity channels between $V_{\mathrm{LSR}}=10$ and $17 \mathrm{~km} \mathrm{~s}^{-1}$. The beam is given in the lower left corner of each panel. A discussion of the joint deconvolution combination method can be found in Section 2.2.

dish map allows us to more accurately calculate outflow mass, momentum, and energy without filtering out large-scale outflow structure.

The combination of interferometer and single dish $\mathrm{CO}$ observations, or the short spacing correction, was carried out according to the non-linear joint deconvolution method of Stanimirovic (2002). This method is particularly useful for recovering flux in mosaics that cover regions with clumpy structure. The combination was done with MIRIAD. First, we Hanning smoothed the single dish map to a similar velocity resolution as the interferometer map, and then regridded the single dish map using the interferometer map as the template. Deconvolution was done with mosmem, using the dirty interferometer map and single dish (regridded) map as inputs, along with dirty beams for each. In order to match the units of the interferometer maps, we converted $T_{A}^{*}$ in the single dish maps to units of $\mathrm{Jy}_{\text {beam }}{ }^{-1}$ using factors of 21.81 and 21.85 for ${ }^{12} \mathrm{CO}$ and ${ }^{13} \mathrm{CO}$, respectively, and assuming an antenna efficiency of 0.47 . In these units and with the same velocity resolution as the interferometer map we measured an rms in the single dish maps of $5 \mathrm{Jy} \mathrm{beam}^{-1}$ and $2 \mathrm{Jy}_{\text {beam }}{ }^{-1}$ per channel for ${ }^{12} \mathrm{CO}$ and ${ }^{13} \mathrm{CO}$, respectively. For the interferometer maps, we used the beam size of the CARMA continuum map, which was only slightly larger than that of the ${ }^{12} \mathrm{CO}$ and ${ }^{13} \mathrm{CO}$ maps, so that the maps would have equivalent resolution for continuum subtraction and for later calculations (see Section 3.4). 
The joint deconvolution was done with the MIRIAD task mosmem. We use the "gull" entropy measure, rather than the cornwell (or maximum emptiness criteria) measure, so as not to force only positive sky-flux solutions and miss negative flux such as absorption below continuum level. In addition, with mosmem we need to specify several parameters so that the convolution process converges within a reasonable number of iterations, including rms factors for both the interferometer map and single dish map (called "rmsfac" in MIRIAD), and the flux calibration factor between the interferometer and single dish map (called "factor" in MIRIAD). The factor "rmsfac" is the ratio between the theoretical rms calculated by MIRIAD and the true rms noise, and it is necessary in order for the task to reduce the residuals (i.e., the difference between the dirty image and the model modified by the point spread function) to have the same rms as the theoretical rms multiplied by the "rmsfac." We used "rmsfac" of 1.6 and 1.4 for the interferometer maps of ${ }^{12} \mathrm{CO}$ and ${ }^{13} \mathrm{CO}$, respectively.

The flux calibration factor is the factor by which the single dish data is multiplied in order to convert it to the same scale as the interferometer data. We determined this factor using the task immerge, choosing uv data in the range 7.2-12 $\mathrm{m}$ (which is the range of "baselines" that the interferometer and single dish data have in common), and specifying channels where emission structure is comparable in the interferometer and singe dish maps. This range of baselines corresponds to $3-5 \mathrm{k} \lambda$. We used a factor of 1.1 for the ${ }^{12} \mathrm{CO}$ deconvolution, and 0.9 for the ${ }^{13} \mathrm{CO}$ deconvolution.

Using these factors, the deconvolution converged within 200 iterations for each channel. The data cubes were then restored with a Gaussian beam. We note that a joint deconvolution was not performed to combine the CARMA continuum map with single-dish data, since we did not have the appropriate single-dish continuum data available. Finally, we subtracted the CARMA-only continuum image from the joint deconvolution molecular line maps using avmaths in the image domain. The resulting mean rms sensitivity of the combined CARMA and FCRAO maps of ${ }^{12} \mathrm{CO}$ and ${ }^{13} \mathrm{CO}$ were 0.27 and $0.14 \mathrm{Jy} \mathrm{beam}^{-1}$ respectively, per $0.3 \mathrm{~km} \mathrm{~s}^{-1}$ channel.

\section{RESULTS}

\subsection{Continuum Sources}

In our map we detect strong continuum sources in the regions SVS 13, IRAS 2, and IRAS 4 (see Figures 1 and 3), with locations, sizes, peak and total fluxes, and masses of each source given in Table 3. Alternate names and references for these sources in the literature are included in Table 2. Continuum emission likely comes from dust in the circumstellar disks and the protostellar envelopes, and this emission pinpoints the positions of protostars that may, although do not necessarily, drive outflows. With continuum map rms of $2 \mathrm{mJy} \mathrm{beam}^{-1}$ and beam size of $6^{\prime \prime} .5 \times 5^{\prime \prime} .3$, we detect nine continuum sources at a greater than $4 \sigma$ level, of which we determine that six are driving outflows (some possibly drive multiple outflows).

With this sensitivity, we are able to detect at greater than a $4 \sigma$ level envelope masses greater than $0.024 M_{\odot}$ for sources within the region of best sensitivity, according to the following mass relation (see Schnee et al. 2010):

$$
M=\frac{d^{2} S_{v}}{B_{v}\left(T_{D}\right) \kappa_{v}},
$$

where $d$ is distance to the source, $S_{v}$ is the $2.7 \mathrm{~mm}$ continuum flux, and $B_{v}\left(T_{D}\right)$ is the Planck function. We assume a dust temperature of $T_{D}=30 \mathrm{~K}$ (Chen et al. 2013), and a dust opacity of $\kappa_{v}=0.1(v / 1200 \mathrm{GHz}) \mathrm{cm}^{2} \mathrm{~g}^{-1}$, which corresponds to $\kappa_{v}=0.009 \mathrm{~cm}^{2} \mathrm{~g}^{-1}$ at $\lambda=2.7 \mathrm{~mm}$, following Looney et al. (2000). We note that dust temperature and opacity in particular contribute to the uncertainty of the masses calculated from continuum. Nonetheless, our mass results reported in Table 3 are consistent within a factor of a few with those of other works based on interferometer observations (Looney et al. 2000; Choi 2001; Chen et al. 2009, 2013), correcting for assumed distance, and other parameters described above.

Following Looney et al. (2000), we acknowledge that the best estimates of masses require detailed modeling, which we do not perform here. We must consider that the integrated flux we measure is likely correlated with beam size and box size, as noted by Ladd et al. (1991). Looney et al. (2000) present this effect for several of the sources that we present here, finding that for these sources the integrated flux differs by a factor of about two to three depending on the box size they choose (see their Table 2). For clarity, we provide in Table 3 the size of the box we defined around each continuum source. We chose box sizes to include all significant emission surrounding the point sources, without including nearby sources. As a simple test, we calculated integrated flux for several of the relatively more isolated sources (IRAS 2A, IRAS $2 \mathrm{~B}$ and IRAS 4A) using a larger box size, and we find very little effect on the results in these cases. For other more closely-spaced objects (i.e., the SVS 13 region, see Section 3.1.1), some of which may perhaps share a common envelope, expanding the box size clearly has an effect if extended emission is more significant.

It is likely that some sources below the detection limit of our continuum observations also drive outflows, such as the candidate outflows presented in Section 3.3. In particular, we do not detect significant continuum emission at the locations of the sources SK 1 and SK 14 (Sandell \& Knee 2001), which are compact $450 \mu \mathrm{m}$ and $850 \mu \mathrm{m}$ sources with mass estimates of $0.07 M_{\odot}$ and $0.01 M_{\odot}$, respectively, according to Sandell \& Knee (2001), but which drive candidate outflows (see Section 3.3). We do not detect the continuum source SK 14 because it has a mass which is below the detection limit of our map. We suspect that we also do not detect the continuum source SK 1 because it lies at the southern edge of our map, where the $4 \sigma$ sensitivity is $0.054 \mathrm{mJy}^{\text {beam }}{ }^{-1}$, which translates to a mass detection limit of about $0.06 M_{\odot}$ (using the same assumptions as above), and the previously reported mass of this object is very close to this limit. In Figure 3 we feature regions where we detected significant continuum emission in our map. In the following subsections, we describe characteristics of the individual continuum sources, as a preface to the discussion of outflow characteristics in Sections 3.2 and 3.3.

Further, we note that three starless cores are located in the mapped region (Bolo 44, SK 16, and HRF 59; see Table 2, as well as Hatchell et al. 2007b), but we do not detect significant continuum emission at any of their locations. SK $16\left(M_{\mathrm{env}}=\right.$ $\left.8.4 M_{\odot}\right)$, located southeast of SVS 13, may have comparable mass to the lowest-mass cores in our map. However, Schnee et al. (2012) suggest that starless cores may not be detected by interferometer observations if their density distribution is sufficiently flat and their continuum emission smooth, and this is very likely the case for SK 16. The other two starless cores (Bolo 44 and HRF 59) lie in the very low sensitivity ( $1 \sigma \mathrm{rms}$ of 

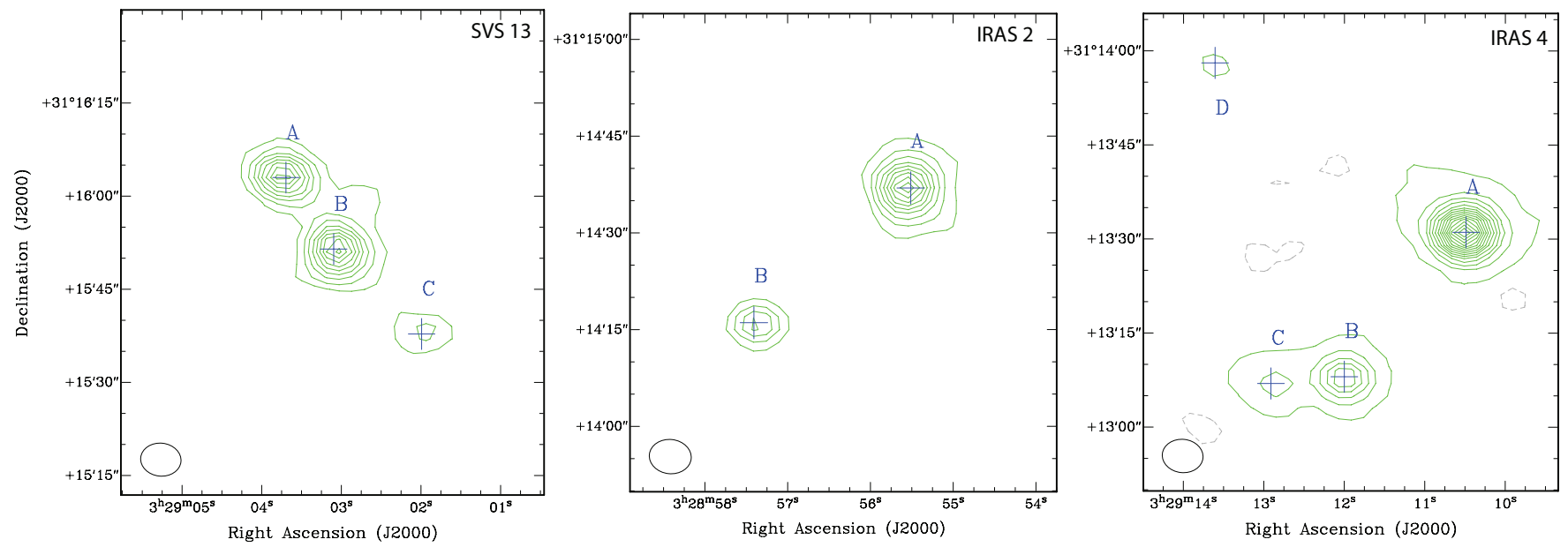

Figure 3. Continuum emission at $112.7 \mathrm{GHz}$ in the regions of SVS13, IRAS2, and IRAS4 (regions are outlined in Figure 1). Green contours begin at $4 \sigma$ with increments of $4 \sigma$ for SVS13 and IRAS2, and increments of $20 \sigma$ for IRAS4, which has the brightest continuum sources in the map. Gray dashed contours show negative intensity measured at the level of $4 \sigma$, which only appears in the region of IRAS 4 , where the strongest continuum sources are found. The rms of the continuum map is $0.002 \mathrm{Jy}$ beam $^{-1}$. The synthesized beam is given in lower left corner of each panel.

(A color version of this figure is available in the online journal.)

$12 \mathrm{mJy}$ beam $^{-1}$ ) region of our map and we do not detect them as their intensity is lower than our $4 \sigma$ sensitivity limits.

\subsubsection{SVS 13 Region}

Within the region shown in the left panel of Figure 3 lies the near-IR source discovered by Strom et al. (1976) known as SVS 13, and later observed with IRAS by Jennings et al. (1987) (i.e., NGC 1333 - IRAS 3), which is responsible for powering $\mathrm{HH}$ 7-11 and the corresponding molecular outflow (see Section 3.2.1). The millimeter continuum observations by Chini et al. (1997) showed three distinct sources in the region-which they name MMS1, MMS2, and MMS3 and are more commonly known as SVS 13A, SVS 13B, and SVS 13C. Of these, SVS 13A corresponds to the optical/NIR source SVS 13. Sensitive, high-angular resolution VLA observations at $3.6 \mathrm{~cm}$ and $6 \mathrm{~cm}$ (Rodríguez et al. 1997, 1999) showed that the millimeter sources correspond to radio sources (VLA 4, 17 and 2), and they detected a fourth source (VLA 3), southwest of SVS 13A. In addition, SVS 13C is associated with an $\mathrm{H}_{2} \mathrm{O}$ maser source known as $\mathrm{H}_{2} \mathrm{O}(\mathrm{B})$ (Haschick et al. 1980; Hirota et al. 2008). Looney et al. (2000) observed the $2.7 \mathrm{~mm}$ continuum emission using BIMA and detected four sources in this region, including SVS 13B, SVS 13C, and resolving SVS 13A into the two components SVS 13A1 and SVS 13A2 (at the position of VLA 3) separated by 6". Chen et al. (2009) suggest that VLA 3 (their naming convention for the source SVS 13A2) in fact forms a protobinary system with SVS 13B based on the velocity field deduced from IRAM Plateau de Bure Interferometer observations, even though VLA 3 appears to be nearer to SVS 13A than SVS 13B as seen projected on the plane of the sky. Encompassing these sources is a large-scale common envelope which was detected with single-dish mm/sub-mm observations (Chini et al. 1997; Chandler \& Richer 2000), but resolved out with interferometer observations.

In this region, we detect the three distinct continuum point sources SVS 13A, SVS 13B, and SVS 13C shown in Figure 3. We cannot resolve VLA 3, but we suggest that the elongated emission near SVS 13A and SVS 13B is likely related to this source. Based on our continuum map, sources SVS 13B and SVS 13C lie southwest of SVS 13A at distances $15^{\prime \prime}(0.02 \mathrm{pc})$ and $36^{\prime \prime}(0.04 \mathrm{pc})$, respectively, along a line with position angle $230^{\circ}$ (see Figure 3). Most studies classify SVS 13 A as a Class I source (e.g., Hatchell et al. 2007b; Evans et al. 2009). Based on its bolometric temperature $(\sim 114 \mathrm{~K})$ and ratio of sub-mm to bolometric luminosity ( $\sim 0.8 \%$ ), Chen et al. (2009) also suggest SVS 13A is Class I, but they note that its association with a cm source and its high-velocity outflow are characteristic of a younger, Class 0 object. Hence, they speculate that SVS 13 A could be a transition Class $0 /$ I source (i.e., a very young Class I source). Both SVS 13B and SVS 13C have been shown to be Class 0 sources based on their spectral energy distributions (SEDs) and $\mathrm{mm} / \mathrm{sub}-\mathrm{mm}$ envelope properties (Chandler \& Richer 2000; Chen et al. 2009).

\subsubsection{IRAS 2 Region}

IRAS 2 was discovered as a protostellar candidate based on IRAS observations by Jennings et al. (1987), and two counterpart sources have been observed at radio, sub-mm, and mm wavelengths (Rodríguez et al. 1999; Sandell et al. 1994; Looney et al. 2000). The northwest source is known as IRAS 2A, and the southeast source is IRAS 2B (see Figure 3). The near-IR source SVS 19 is very nearly coincident with IRAS 2B; however, Rodríguez et al. (1999) suggest that SVS 19 corresponds to the bright G2 IV star in the foreground, BD $+30^{\circ} 547$ (a.k.a. ASR 130). In addition to IRAS $2 A$ and IRAS 2B, Sandell \& Knee (2001) detected the source IRAS 2C northwest of IRAS $2 A$.

In our map we detect two continuum sources separated by $35^{\prime \prime}(0.04 \mathrm{pc})$ in the IRAS 2 region, shown in Figure 3 and previously detected by Looney et al. (2000). IRAS 2A is a Class 0 protostar, while IRAS $2 \mathrm{~B}$ is likely a young Class I source, based on its bolometric temperature $\left(T_{\text {bol }}=100\right)$ and spectral index ( $\alpha=1.48)$ (Evans et al. 2009). However, Sandell $\&$ Knee (2001) called IRAS 2B a possible Class 0 source, and Gutermuth et al. (2008) classify it as deeply embedded. Since IRAS 2A drives nearly perpendicular outflows, described in more detail in Section 3.2.2, it is likely that this is a binary source (Chen et al. 2013 and references therein), although we detect only one source. Chen et al. (2013) report a separation between the components of IRAS 2A of 1".5 and a mass ratio of 0.05 according to Submillimeter Array $850 \mu \mathrm{m}$ dust continuum results. 
Table 2

YSOs and Starless Cores within the Mapped Region

\begin{tabular}{|c|c|c|c|c|}
\hline Source & $\alpha(\mathrm{J} 2000)$ & $\delta(\mathrm{J} 2000)$ & Class $^{\mathrm{a}}$ & Other Names ${ }^{b}$ \\
\hline \multicolumn{5}{|l|}{ Classes S, 0, I } \\
\hline IRAS 5 & $03: 28: 43.27$ & $31: 17: 33.10$ & I & SVS 9, ASR 126, [GMM2008] 18, SSTc2d J032843.28+311732.9 \\
\hline ASR 127 & 03:28:43.56 & $31: 17: 36.50$ & I & {$[$ GMM2008] 45, Bolo 31(I) } \\
\hline ASR 41 & 03:28:51.27 & $31: 17: 39.50$ & I & {$[$ GMM2008] 20, SSTc2d J032851.26+311739.3 } \\
\hline IRAS $2 \mathrm{~A}$ & $03: 28: 55.30$ & $31: 14: 36.40$ & $0^{\mathrm{d}}$ & $\begin{array}{l}\text { VLA 7, SK 8, HRF 44(0), [GMM2008] 21(I), Bolo 38(0) }{ }^{\mathrm{c}}, \text { SSTc2d } \\
\text { J032855.55+311436.7(0) }\end{array}$ \\
\hline IRAS 2B & $03: 28: 57.37$ & $31: 14: 16.20$ & $I^{\mathrm{d}}$ & $\begin{array}{l}\text { SVS } 19^{f}, \text { VLA 10, SK 7, [GMM2008] 3(0), Bolo 38(I) }{ }^{\mathrm{c}}, \text { SSTc2d } \\
\text { J032857.36+311415.9(I) }\end{array}$ \\
\hline SK 1 & 03:29:00.40 & $31: 12: 01.50$ & 0 & HRF 65, Bolo 41, [GMM2008] 4, SSTc2d J032900.55+311200.8 \\
\hline SVS $13 \mathrm{C}$ & 03:29:01.97 & $31: 15: 37.40$ & 0 & MMS3, VLA 2, SK 11, $\mathrm{H}_{2} \mathrm{O}(\mathrm{B})$ \\
\hline VLA 3 & 03:29:03.00 & $31: 16: 02.00$ & 0 & \\
\hline SVS 13B & 03:29:03.05 & $31: 15: 52.50$ & 0 & MMS2, VLA 17, SK 12 \\
\hline SVS 13A & 03:29:03.20 & $31: 15: 59.00$ & I & $\begin{array}{l}\text { SVS 13, IRAS 3, ASR 1, MMS1, VLA 4, SK 13, HRF 43, [GMM2008] } \\
\text { 29, Bolo 43, SSTc2d J032903.78+311603.8 }\end{array}$ \\
\hline SK $14^{\mathrm{g}}$ & 03:29:03.70 & $31: 14: 53.10$ & 0 & VLA 19, HRF 52, [GMM2008] 5, Bolo 46, SSTc2d J032904.06+311446.5 \\
\hline Bolo 44 & 03:29:04.90 & $31: 18: 41.20$ & S & \\
\hline SK 15 & 03:29:06.50 & $31: 15: 38.60$ & I & HRF 50 \\
\hline SK 18 & 03:29:07.10 & $31: 17: 23.70$ & 0 & HRF 62 \\
\hline SK 16 & 03:29:08.80 & $31: 15: 18.10$ & S & HRF 51 \\
\hline IRAS 4A & 03:29:10.40 & $31: 13: 30.00$ & 0 & $\begin{array}{l}\text { VLA 25, SK 4, HRF 41, [GMM2008] 6, Bolo 48c }, \text { SSTc2d } \\
\text { J032910.49+311331.0 }\end{array}$ \\
\hline SSTc2d J032910.65+311340.0 & 03:29:10.65 & $31: 13: 40.00$ & $\mathrm{I}$ & \\
\hline SK 20 & 03:29:10.71 & $31: 18: 21.20$ & $\mathrm{I}^{\mathrm{d}}$ & Bolo 49(0) ${ }^{\mathrm{c}}, \mathrm{SSTc} 2 \mathrm{~d}$ J032910.68+311820.6(I) \\
\hline IRAS 7 & 03:29:11.00 & $31: 18: 27.40$ & $\mathrm{I}^{\mathrm{d}}$ & HRF 46(0), [GMM2008] 35(I), SSTc2d J032910.99+311826.0(I) \\
\hline SK 21 & 03:29:11.25 & $31: 18: 31.70$ & $\mathrm{I}^{\mathrm{d}}$ & $\begin{array}{c}\text { ASR 33, VLA 27, [GMM2008] 7(0), Bolo 49(0) }{ }^{\mathrm{c}}, \text { SSTc2d } \\
\text { J032911.26+311831.4(I) }\end{array}$ \\
\hline IRAS 4B & 03:29:12.00 & $31: 13: 10.00$ & 0 & $\begin{array}{l}\text { VLA 28, SK 3, HRF 42, [GMM2008] 8, Bolo } 48^{\mathrm{c}}, \text { SSTc2d } \\
\text { J032912.06+311305.4 }\end{array}$ \\
\hline IRAS 4C & 03:29:12.88 & 31:13:08.20 & 0 & SK 2 \\
\hline ASR 30 & 03:29:12.95 & $31: 18: 14.60$ & I & [GMM2008] 36, Bolo 49(I) ${ }^{\mathrm{c}}$, SSTc2d J032912.97+311814.3 \\
\hline IRAS 4D & 03:29:13.60 & $31: 13: 55.00$ & 0 & $\begin{array}{l}\text { VLA 29, SK 5, HRF 48, [GMM2008] 9, Bolo 48c , SSTc2d } \\
\text { J032913.54+311358.2 }\end{array}$ \\
\hline HRF 59 & 03:29:16.50 & $31: 12: 34.60$ & $\mathrm{~S}$ & \\
\hline \multicolumn{5}{|l|}{ Classes F, II, III } \\
\hline SSTc2d J032846.21+311638.4 & 03:28:46.19 & $31: 16: 38.70$ & III & SVS 21, ASR 128, [GMM2008] 47 \\
\hline SSTc2d J032847.84+311655.1 & 03:28:47.82 & $31: 16: 55.30$ & II & SVS 17, ASR 111, [GMM2008] 49 \\
\hline ASR 67 & $03: 28: 48.76$ & $31: 16: 08.90$ & $\mathrm{~F}^{\mathrm{d}}$ & [GMM2008] 19(I), SSTc2d J032847.84+311655.1(F) \\
\hline SSTc2d J032851.03+311818.5 & 03:28:51.02 & $31: 18: 18.50$ & II & SVS 10, ASR 122, [GMM2008] 50 \\
\hline SSTc2d J032851.08+311632.4 & 03:28:51.07 & $31: 16: 32.60$ & II & ASR 44, [GMM2008] 51 \\
\hline SSTc2d J032852.15+311547.1 & $03: 28: 52.13$ & $31: 15: 47.20$ & II & ASR 45, [GMM2008] 53 \\
\hline SSTc2d J032852.92+311626.4 & 03:28:52.90 & $31: 16: 26.60$ & II & ASR 46, [GMM2008] 55 \\
\hline ASR 97 & 03:28:53.58 & $31: 12: 14.70$ & II & [GMM2008] 56 \\
\hline SSTc2d J032853.96+311809.3 & 03:28:53.93 & 31:18:09.30 & II & ASR 40, [GMM2008] 57 \\
\hline SSTc2d J032854.09+311654.2 & 03:28:54.07 & $31: 16: 54.50$ & II & SVS $18^{\mathrm{h}}$, ASR 42, [GMM2008] 58 \\
\hline SSTc2d J032854.63+311651.1 & 03:28:54.61 & $31: 16: 51.30$ & II & SVS $18^{\mathrm{h}}$, ASR 43, [GMM2008] 59 \\
\hline ASR 109 & 03:28:54.92 & $31: 15: 29.20$ & II & [GMM2008] 60 \\
\hline SSTc2d J032855.08+311628.7 & 03:28:55.07 & $31: 16: 28.80$ & II & ASR 107, [GMM2008] 61 \\
\hline ASR 108 & 03:28:55.15 & $31: 16: 24.80$ & II & {$[$ GMM2008] 62} \\
\hline SSTc2d J032856.65+311835.5 & 03:28:56.64 & $31: 18: 35.70$ & II & SVS 11, ASR 120, Bolo 39, [GMM2008] 65 \\
\hline SSTc2d J032856.97+311622.3 & 03:28:56.95 & $31: 16: 22.30$ & II & SVS 15, ASR 118, [GMM2008] 67 \\
\hline SSTc2d J032857.18+311534.6 & 03:28:57.17 & $31: 15: 34.60$ & II & ASR 17, [GMM2008] 68 \\
\hline SSTc2d J032857.21+311419. $1^{\mathrm{i}}$ & 03:28:57.21 & $31: 14: 19.10$ & III & \\
\hline SSTc2d J032858.11+311803.7 & 03:28:58.11 & 31:18:03.70 & III & ASR 36 \\
\hline SSTc2d J032859.32+311548.7 & 03:28:59.32 & $31: 15: 48.50$ & $\mathrm{~F}$ & SVS 16, ASR 106, [GMM2008] 73 \\
\hline SSTc2d J032901.88+311653.2 & 03:29:01.88 & $31: 16: 53.20$ & $\mathrm{~F}$ & ASR 11 \\
\hline ASR 3 & 03:29:02.16 & $31: 16: 11.40$ & II & [GMM2008] 76 \\
\hline ASR 63 & 03:29:03.39 & $31: 18: 40.10$ & II & [GMM2008] 80 \\
\hline SSTc2d J032904.68+311659.0 & 03:29:04.67 & $31: 16: 59.20$ & II & ASR 105, [GMM2008] 84 \\
\hline SSTc2d J032904.73+311134.9 & 03:29:04.73 & $31: 11: 35.00$ & II & ASR 99, [GMM2008] 85 \\
\hline SSTc2d J032905.78+311639.6 & 03:29:05.76 & $31: 16: 39.70$ & II & SVS 14, ASR 7, VLA 22, [GMM2008] 88 \\
\hline SSTc2d J032906.33+311346.4 & 03:29:06.32 & $31: 13: 46.50$ & II & ASR 53, [GMM2008] 89 \\
\hline SSTc2d J032909.40+311413.8 & 03:29:09.41 & $31: 14: 14.10$ & II & ASR 54, [GMM2008] 135 \\
\hline SSTc2d J032910.84+311642.6 & 03:29:10.82 & $31: 16: 42.70$ & II & ASR 23, [GMM2008] 99 \\
\hline
\end{tabular}


Table 2

(Continued)

\begin{tabular}{|c|c|c|c|c|}
\hline Source & $\alpha(\mathrm{J} 2000)$ & $\delta(\mathrm{J} 2000)$ & Class $^{\mathrm{a}}$ & Other Names ${ }^{\mathrm{b}}$ \\
\hline SSTc2d J032912.06+311301.7 & 03:29:12.06 & 31:13:01.70 & $\mathrm{F}$ & \\
\hline ASR 28 & 03:29:13.04 & $31: 17: 38.40$ & II & [GMM2008] 105 \\
\hline SSTc2d J032914.40+311444.1 & 03:29:14.40 & $31: 14: 44.10$ & II & \\
\hline
\end{tabular}

Notes.

${ }^{a}$ Classifications as starless (S), Class 0, Class I, flat-SED (F), Class II or Class III, where information is available in the literature.

b Prefixes and associated references: "SVS"-Strom et al. (1976); "IRAS"-Jennings et al. (1987); "ASR"-Aspin et al. (1994); "MMS"-Chini et al. (1997); "VLA"-Rodríguez et al. (1997, 1999); "SK"—Sandell \& Knee (2001); "HRF"—Hatchell et al. (2007b); [GMM2008]—Gutermuth et al. (2008); "Bolo"—Enoch et al. (2009); "SSTc2d"-Evans et al. (2009).

${ }^{\mathrm{c}}$ More than one protostar associated with a Bolocam source, or source is in a crowded region.

${ }^{\mathrm{d}}$ Source classification differs among references. In these cases, we assume the classification from Evans et al. (2009), but we give the classifications for each reference, where appropriate.

${ }^{\mathrm{e}}$ Classified as Class I based on $\alpha(=0.62)$, but with high $T_{\mathrm{Bol}}(=1000 \mathrm{~K})$.

${ }^{\mathrm{f}}$ Source position is coincident, but Rodríguez et al. (1999) suggest that this is the G2 IV star BD $+30^{\circ} 547$ (=ASR 130).

g Sources from different references within about $8^{\prime \prime}$.

${ }^{\mathrm{h}}$ Source from Strom et al. (1976) is very near to two identified sources such that we cannot distinguish to which it corresponds.

${ }^{i}$ Near IRAS 2B.

j Near IRAS 4C.

Table 3

Continuum Sources

\begin{tabular}{|c|c|c|c|c|c|c|c|c|c|c|}
\hline \multirow[t]{2}{*}{ Source } & \multirow[t]{2}{*}{ Class } & \multicolumn{2}{|c|}{ Position } & \multirow{2}{*}{$\begin{array}{c}\text { Box Size }^{a} \\
\left({ }^{\prime \prime}\right)\end{array}$} & \multicolumn{2}{|c|}{ Object Size } & \multirow{2}{*}{$\begin{array}{l}\text { PA } \\
\left({ }^{\circ}\right)\end{array}$} & \multirow{2}{*}{$\begin{array}{l}\text { Peak Intensity } \\
\left(\mathrm{mJy} \mathrm{beam}^{-1}\right)\end{array}$} & \multirow{2}{*}{$\begin{array}{c}\text { Total Flux } \\
\text { (mJy) }\end{array}$} & \multirow{2}{*}{$\begin{array}{l}\text { Mass }^{\mathrm{b}} \\
\left(M_{\odot}\right) \\
\end{array}$} \\
\hline & & $\alpha(\mathrm{J} 2000)$ & $\delta(\mathrm{J} 2000)$ & & Major (") & Minor (") & & & & \\
\hline SVS 13A & I & 03:29:03.7 & $+31: 16: 03.1$ & $20 \times 16$ & $9 \pm 1$ & $6 \pm 1$ & $67 \pm 12$ & $65 \pm 6$ & 106 & 0.3 \\
\hline SVS 13C & 0 & 03:29:01.9 & $+31: 15: 38.6$ & $18 \times 10$ & $11 \pm 2$ & $7 \pm 1$ & $85 \pm 14$ & $16 \pm 3$ & 35 & 0.09 \\
\hline IRAS $2 \mathrm{~A}$ & 0 & $03: 28: 55.5$ & $+31: 14: 37.0$ & $22 \times 22$ & $8 \pm 0.5$ & $8 \pm 0.5$ & $51 \pm 117$ & $68 \pm 4$ & 129 & 0.3 \\
\hline IRAS 2B & I & $03: 28: 57.4$ & $+31: 14: 15.9$ & $14 \times 12$ & $7 \pm 0.3$ & $5 \pm 0.3$ & $87 \pm 9$ & $35 \pm 2$ & $\ldots{ }^{c}$ & $0.09^{c}$ \\
\hline IRAS 4A & 0 & $03: 29: 10.5$ & $+31: 13: 31.1$ & $26 \times 26$ & $7 \pm 0.2$ & $6 \pm 0.1$ & $84 \pm 5$ & $580 \pm 12$ & 773 & 2.0 \\
\hline IRAS 4D & 0 & $03: 29: 13.6$ & $+31: 13: 57.9$ & $10 \times 10$ & $6 \pm 2$ & $4 \pm 1$ & $65 \pm 20$ & $20 \pm 5$ & $\ldots{ }^{c}$ & $0.05^{\mathrm{c}}$ \\
\hline
\end{tabular}

Notes.

${ }^{a}$ Box size refers to the region defined around the continuum source, used as input for MIRIAD task imfit and described in Section 3.1.

$\mathrm{b}$ Mass calculation is described in Section 3.1 and follows the method of Looney et al. (2000).

${ }^{c}$ MIRIAD task imfit failed to fit a Gaussian to these sources. For these sources, we find the mass (lower limit) based on peak intensity.

\subsubsection{IRAS 4 Region}

IRAS 4 was also observed in the IR by Jennings et al. (1987), with the three radio counterparts VLA 25, VLA 28, and VLA 29 observed by Rodríguez et al. (1999). VLA 25 and VLA 28 correspond to IRAS 4A and IRAS 4B in Table 2. Sandell \& Knee (2001) resolved VLA 28 into two components, which correspond to the sources commonly known as IRAS 4B and IRAS 4C (these are IRAS 4BW and IRAS 4BE in Sandell \& Knee, 2001). VLA 29 corresponds to the source we call IRAS 4D. ${ }^{8}$

Figure 3 shows the four continuum sources, IRAS 4A, IRAS 4B, IRAS 4C, and IRAS 4D, of which IRAS 4A and IRAS $4 \mathrm{~B}$ are the strongest continuum sources detected in our map. IRAS 4B is located $33^{\prime \prime}(0.04 \mathrm{pc})$ southeast of IRAS 4A, and IRAS $4 \mathrm{C}$ is located $14^{\prime \prime}(0.02 \mathrm{pc})$ east of IRAS 4B. IRAS 4D is a weaker (with a $7 \sigma$ peak) source that lies $54^{\prime \prime}(0.06 \mathrm{pc})$

\footnotetext{
8 This naming convention is chosen to abide by the IRAS 4A, 4B, 4C sequence used by Looney et al. (2000) and Chen et al. (2013), although it should be noted that the source we call IRAS 4D is called IRAS 4C in several other works (Lefloch et al. 1998; Sandell et al. 1991), and the sources we call IRAS 4B and 4C are called, e.g., IRAS 4BW/E (Sandell \& Knee 2001) or IRAS4BI/II (Choi 2001).
}

northeast of IRAS 4A. The four IRAS 4 sources have been classified as Class 0 (Sandell \& Knee 2001; Hatchell et al. 2007b; Hatchell \& Dunham 2009; Evans et al. 2009). High angular-resolution continuum observations have shown that IRAS 4A is a binary source (Lay et al. 1995; Looney et al. 2000; Chen et al. 2013) whose components have a separation of 1."4 and mass ratio of 0.5 . Given the angular resolution of our CARMA observations we cannot resolve the components that make up IRAS 4A in our map. Previous works have also shown that IRAS 4B and IRAS 4C are components of a protostellarmultiple system (Lay et al. 1995; Looney et al. 2000; Chen et al. 2013).

\subsection{Molecular Outflow Emission}

Here we present CO (1-0) outflows associated with SVS 13, IRAS 2, and IRAS 4, followed by several outflow candidates in Section 3.3. We also compare with previous observations of molecular outflows across this region, including singledish maps of CO (1-0) by Knee \& Sandell (2000) and CO (3-2) by Knee \& Sandell (2000) and Curtis et al. (2010a, 
2010b), ${ }^{9}$ as well as interferometry studies of individual outflows within the region, mentioned more specifically in the following subsections.

Our map of ${ }^{12} \mathrm{CO}$ outflow emission is shown in Figure 4, with outflows detected within a region spanning $\sim 7^{\prime} \times 7^{\prime}$. These cool outflows are traced well with ${ }^{12} \mathrm{CO}$, which probes the entrained molecular gas and allows us to determine physical outflow properties. In these regions, the ${ }^{13} \mathrm{CO}$ is less optically thick than the ${ }^{12} \mathrm{CO}$, and it was used to make a reliable estimate of the outflow mass (see Section 3.4). Table 4 gives physical traits of the outflows, including position angles and sizes. In the following subsections we describe their distinct morphologies in more detail. Table 5 gives quantitative characteristics including mass, momentum, and energy for the outflows in the region, as well as the outflows' driving sources. The method to calculate mass, momentum, and energy is described in Section 3.4.

Our full velocity coverage was $v_{\mathrm{LSR}}=-2.3$ to $17.3 \mathrm{~km} \mathrm{~s}^{-1}$, and we group channels as blue high-velocity $v_{\mathrm{LSR}}=-2.3$ to $-0.4 \mathrm{~km} \mathrm{~s}^{-1}$, blue medium-velocity $v_{\mathrm{LSR}}=-0.1$ to 3.1 , blue low-velocity $v_{\mathrm{LSR}}=3.4$ to $5.9 \mathrm{~km} \mathrm{~s}^{-1}$, red low-velocity $v_{\mathrm{LSR}}=9.7$ to $11.0 \mathrm{~km} \mathrm{~s}^{-1}$, red mid-velocity $v_{\mathrm{LSR}}=11.3$ to $14.5 \mathrm{~km} \mathrm{~s}^{-1}$, and red high-velocity $v_{\mathrm{LSR}}=14.8$ to $17.3 \mathrm{~km} \mathrm{~s}^{-1}$, chosen to show the predominant outflow features. Here we assume a cloud velocity of $v_{\text {cloud }}=8 \mathrm{~km} \mathrm{~s}^{-1}$, in agreement with the velocity reported by Curtis et al. (2010b). Throughout, we will use the notation of $v_{\text {LSR }}$ to indicate channel velocity (relative to local standard of rest), cloud velocity $v_{\text {cloud, }}$ and outflow velocity $v_{\text {out }}=v_{\text {LSR }}-v_{\text {cloud }}$ (along the line of sight).

\subsubsection{SVS 13A Outflow}

The most prevalent outflow in our map is SVS 13A, shown as Region I of Figure 4 and featured in Figure 5. Wide-angle blueshifted and redshifted outflow lobes are oriented SE-NW and intersect at the location of the young Class I source SVS 13A, which was also suggested by Knee \& Sandell (2000) to be the driving source of this outflow. In most channels, we see the morphology of each lobe as a "donut"-shape, with strong emission around the edges, and a cavity without emission at the center of each lobe. Near the driving source SVS 13A, blueshifted and redshifted emission from the SE and NW lobes coincide along the line of sight, in agreement with a moderate inclination angle (e.g., $40^{\circ}$ with respect to the line of sight, Davis et al. 2011). We note that some of the lack of emission detected in the center of the outflow shells is likely due to destructively interfering negative side-lobes (i.e., not all flux is fully recovered in our maps). The outflow emission from this source has a broad morphology, with blueshifted and redshifted lobes having opening angles of $96^{\circ}$ and $76^{\circ}$ and collimation factors of 0.7 and 0.4 , respectively. This wide-angle morphology is consistent with other outflows powered by Class I protostars (e.g., Arce \& Sargent 2006).

Within our spectral coverage, the morphology of the outflow appears to be velocity dependent, with a more clumpy structure seen in the high-velocity channels, and a smoother shell-like structure seen in low-velocity channels. The low-velocity outflow morphology is reminiscent of L1551, for which MoriartySchieven et al. (1987) suggested that the low-velocity outflow gas is entrained as an expanding shell at the outflow cavity edges, while higher-velocity outflow gas is more concentrated

\footnotetext{
9 The maps of Knee \& Sandell (2000) are $\sim 63 \operatorname{arcmin}^{2}$, and the survey was later expanded upon by Hatchell et al. (2007a) and Hatchell \& Dunham (2009). Curtis et al. (2010a, 2010b) mapped a region $612 \operatorname{arcmin}^{2}$ in NGC 1333.
}

along the outflow axis. In the SVS 13A outflow, Bachiller et al. (2000) previously identified high-velocity "molecular bullets" of $\mathrm{CO}(2-1)$ emission at velocities greater than about $75 \mathrm{~km} \mathrm{~s}^{-1}$ from the cloud velocity, and as expected we detect emission over the full extent of our velocity coverage (approximately $v_{\text {cloud }} \pm 10 \mathrm{~km} \mathrm{~s}^{-1}$ ). Our limited bandwidth does not allow us to detect the high-velocity $\left(\left|v_{\text {out }}\right|>10 \mathrm{~km} \mathrm{~s}^{-1}\right)$ emission for this outflow, and therefore our mass, momentum, and kinetic energy estimates for the SVS 13A outflow should be considered as lower limits. We see strong "clumpy" emission features especially in the southeast lobe, at the locations marked F1, F2 and F3 in Figure 5, where F1 and F2 show strong (up to $\sim 60 \sigma$ ) blueshifted emission at $V_{\mathrm{LSR}}=3.1-4.6 \mathrm{~km} \mathrm{~s}^{-1}$, and F3 shows strong $(>40 \sigma)$ redshifted emission at $V_{\mathrm{LSR}}=10.3-10.7 \mathrm{~km} \mathrm{~s}^{-1}$, as well as less-intense blueshifted emission. These are the strongest, most concentrated $\mathrm{CO}$ emission clumps in our map.

Comparing our map with previous millimeter interferometric observations of this source (e.g., Bachiller et al. 2000), it can be seen that fully mapping the outflow area and velocity extent is necessary to reveal the complex morphology of this source (and of other wide-angle outflows). With our spatial coverage we recover the full shell-like structure of both outflow lobes, which were previously only partially seen by Bachiller et al. (2000), and we clearly see that these lobes intersect at the position of the source SVS 13A.

Spitzer/IRAC Band 2 observations of this region (Gutermuth et al. 2008) reveal strong knotty $4.5 \mu \mathrm{m}$ emission, which is thought to arise from shock-heated molecular hydrogen (e.g., Neufeld \& Yuan 2008). In the SVS 13A outflow, the bright IRAC 2 emission likely indicates the region where the protostellar wind collides with ambient cloud material, particularly near the SE and NW apexes of the outflow lobes. Apart from the main contiguous SE-NW CO outflow lobes, there are two distinct "blobs" of ${ }^{12} \mathrm{CO}$ emission to the southeast of the southern lobe (clump F3, previously mentioned) and northwest of the northern lobe (clump F4). The clumpy morphology of the outflow and the fact that different ${ }^{12} \mathrm{CO}$ outflow blobs are coincident with discrete shock-induced $\mathrm{H}_{2}$ knots are all consistent with this being an episodic outflow, similar to the HH 315 molecular outflow (Arce \& Goodman 2002a, 2002b).

We calculate masses of the SE (blue) and NW (red) outflow lobes to be $1 M_{\odot}$ and $0.7 M_{\odot}$, spanning distances of $2^{\prime} .0(0.14 \mathrm{pc})$ and 2:7 (0.19 pc) and areas of 2.0 and $2.2 \operatorname{arcmin}^{2}\left(\sim 0.01 \mathrm{pc}^{2}\right)$ on the plane of the sky, respectively. It has previously been noted that additional outflows may exist near this region (e.g., Davis et al. 2008; Knee \& Sandell 2000; Curtis et al. 2010b), but may be confused by the stronger SE-NW outflow. In Section 3.3.1 we describe emission which may pertain to an outflow driven approximately south-north by SVS 13C.

\subsubsection{IRAS 2 Outflows}

We detect three outflows in the IRAS 2 region, where the Class 0 source IRAS $2 \mathrm{~A}$ and the Class I source IRAS 2B reside (see Figure 6). Of the outflows associated with IRAS 2A, one outflow is very collimated and oriented approximately west-east (hereafter IRAS 2A west-east), and the other shows a wide-angle morphology oriented approximately south-north (hereafter IRAS 2A south-north). The shell-like IRAS 2A south-north outflow was previously observed by Liseau et al. (1988), and the "jet-like" IRAS 2A west-east outflow was observed by Sandell et al. (1994) and Bachiller et al. (1998). Knee \& Sandell (2000) suggested that IRAS 2A drives both, nearly orthogonal outflows. Curtis et al. (2010b) also detect 
Table 4

Outflow Lobe Physical Traits

\begin{tabular}{|c|c|c|c|c|c|c|c|c|c|c|c|c|c|c|c|c|c|c|c|c|c|}
\hline \multirow[b]{2}{*}{ Name } & \multicolumn{7}{|c|}{ Blue Lobe } & \multicolumn{7}{|c|}{ Red Lobe } & \multicolumn{5}{|c|}{ Blue and Red Average } & \multicolumn{2}{|c|}{ Sum } \\
\hline & $\begin{array}{c}\text { PA } \\
\text { (deg) }\end{array}$ & $\begin{array}{c}a \\
\left({ }^{\prime \prime}\right)\end{array}$ & $\begin{array}{c}b \\
\left({ }^{\prime \prime}\right)\end{array}$ & $\begin{array}{l}\text { Coll. }^{\mathrm{a}} \\
(a / b)\end{array}$ & $\begin{array}{c}\theta^{\mathrm{b}} \\
(\mathrm{deg})\end{array}$ & $\begin{array}{c}A^{\mathrm{c}} \\
\left(10^{3} \operatorname{arcsec}^{2}\right)\end{array}$ & $\begin{array}{c}V^{\mathrm{c}} \\
\left(10^{-4} \mathrm{pc}^{3}\right)\end{array}$ & $\begin{array}{c}\text { PA } \\
(\operatorname{deg})\end{array}$ & $\begin{array}{c}a \\
\left({ }^{\prime \prime}\right)\end{array}$ & $\begin{array}{c}b \\
\left({ }^{\prime \prime}\right)\end{array}$ & $\begin{array}{l}\text { Coll. } \\
(a / b)\end{array}$ & $\begin{array}{c}\theta \\
(\mathrm{deg})\end{array}$ & $\begin{array}{c}A \\
\left(10^{3} \operatorname{arcsec}^{2}\right)\end{array}$ & $\begin{array}{c}V \\
\left(10^{-4} \mathrm{pc}^{3}\right)\end{array}$ & $\begin{array}{c}\text { PA } \\
\text { (deg) }\end{array}$ & $\begin{array}{c}a \\
(")\end{array}$ & $\begin{array}{c}b \\
\left({ }^{\prime \prime}\right)\end{array}$ & $\begin{array}{l}\text { coll. } \\
(a / b)\end{array}$ & $\begin{array}{c}\theta \\
(\mathrm{deg})\end{array}$ & $\begin{array}{c}A \\
\left(10^{3} \operatorname{arcsec}^{2}\right)\end{array}$ & $\begin{array}{c}V \\
\left(10^{-4} \mathrm{pc}^{3}\right)\end{array}$ \\
\hline SVS $13 A^{d}$ & 120 & 122 & 82 & 0.7 & 96 & 7.88 & 6.39 & 140 & 163 & 57 & 0.4 & 76 & 7.35 & 4.16 & 130 & 143 & 70 & 0.6 & 86 & 15.23 & 10.55 \\
\hline SVS 13C & 8 & 212 & 86 & 0.4 & 69 & 14.41 & 12.28 & 8 & 180 & 86 & 0.5 & 60 & 12.21 & 10.41 & 8 & 196 & 86 & 0.5 & 65 & 26.63 & 22.68 \\
\hline IRAS 2A west-east & 103 & 108 & 21 & 0.2 & 30 & 1.74 & 0.35 & 105 & 84 & 22 & 0.3 & 32 & 1.48 & 0.33 & 104 & 96 & 22 & 0.3 & 31 & 3.22 & 0.68 \\
\hline IRAS 2A south-north & 24 & 188 & 70 & 0.4 & 56 & $8.71^{\mathrm{e}}$ & $6.56^{\mathrm{e}}$ & 4 & 133 & 90 & 0.7 & 93 & 9.39 & 8.33 & 14 & 161 & 80 & 0.6 & 75 & 18.10 & 14.89 \\
\hline IRAS $2 B^{f}$ & 24 & 181 & 92 & 0.5 & 60 & 12.99 & 11.73 & $\cdots$ & $\cdots$ & $\cdots$ & $\cdots$ & $\cdots$ & $\cdots$ & $\cdots$ & $\cdots$ & $\cdots$ & $\cdots$ & $\cdots$ & $\cdots$ & $\cdots$ & $\ldots$ \\
\hline IRAS $4 A^{g}$ & 35 & 104 & 24 & 0.2 & 100 & 2.00 & 0.48 & 35 & 125 & 29 & 0.2 & 82 & 2.87 & 0.83 & 35 & 115 & 27 & 0.2 & 91 & 4.87 & 1.31 \\
\hline IRAS 4B & 180 & 10 & 10 & 1.0 & 90 & 0.08 & 0.01 & 180 & 11 & 11 & 1.0 & 90 & 0.09 & 0.01 & 180 & 11 & 11 & 1.0 & 90 & 0.17 & 0.018 \\
\hline SK 14 & 145 & 71 & 39 & 0.6 & 93 & 2.16 & 0.83 & 145 & 58 & 35 & 0.6 & 55 & 1.59 & 0.55 & 145 & 65 & 37 & 0.6 & 74 & 3.75 & 1.38 \\
\hline SK 1 & 98 & 165 & 32 & 0.2 & $\ldots$ & $1.63^{\mathrm{h}}$ & $0.58^{\mathrm{h}}$ & 95 & 165 & 32 & 0.2 & & $2.04^{\mathrm{h}}$ & $0.72^{\mathrm{h}}$ & 97 & 165 & 32 & 0.2 & $\ldots$ & 3.67 & 1.3 \\
\hline $\mathrm{C} 1$ & $\cdots$ & $\cdots$ & $\cdots$ & $\cdots$ & $\cdots$ & $\cdots$ & $\cdots$ & 157 & 180 & 36 & 0.2 & $\cdots$ & 5.09 & 1.81 & $\cdots$ & $\cdots$ & $\cdots$ & $\ldots$ & $\cdots$ & $\cdots$ & $\cdots$ \\
\hline $\mathrm{C} 2$ & 148 & 122 & 34 & 0.3 & $\cdots$ & 3.25 & 1.09 & $\cdots$ & $\cdots$ & $\cdots$ & $\cdots$ & $\cdots$ & $\cdots$ & $\cdots$ & $\cdots$ & $\cdots$ & $\cdots$ & $\cdots$ & $\cdots$ & $\cdots$ & $\cdots$ \\
\hline C3 & $\cdots$ & $\cdots$ & $\cdots$ & $\cdots$ & $\cdots$ & $\cdots$ & $\cdots$ & 158 & 202 & 65 & 0.3 & $\cdots$ & 10.26 & 6.56 & $\cdots$ & $\cdots$ & $\ldots$ & $\cdots$ & $\ldots$ & $\cdots$ & $\cdots$ \\
\hline $\mathrm{C} 4$ & $\ldots$ & $\ldots$ & $\ldots$ & $\ldots$ & $\ldots$ & $\ldots$ & $\ldots$ & 160 & 144 & 18 & 0.1 & $\ldots$ & 2.04 & 0.36 & $\ldots$ & $\ldots$ & $\ldots$ & $\ldots$ & $\ldots$ & $\ldots$ & $\ldots$ \\
\hline
\end{tabular}

Notes.

${ }^{\text {a }}$ Collimation factor $b / a$, where $b$ is width and $a$ is length of outflow lobe.

$\mathrm{b}$ Opening angle $\theta$ fit by eye. Fit was only made for outflows with identified sources, and where emission was detected near the identified source.

${ }^{c}$ Area $(A)$ and volume $(V)$ of ellipse fit to outflow lobe, assuming idealized, symmetric outflow geometry, as in Offner et al. (2011). For volume, we assume that depth equals width of outflow lobe $(c=b)$.

$\mathrm{d}$ "Blue" lobe of SVS 13A is in southeast, and includes red channels $9.08-11.30 \mathrm{~km} \mathrm{~s}^{-1}$, in addition to blue channels.

e Area and volume of IRAS 2A-south reported after subtracting area and volume of SK 1-west, where the two lobes overlap. See Section 3.2.2 for more discussion.

${ }^{\mathrm{f}}$ We did not disentangle emission from nearby contaminating outflows to estimate mass for IRAS 2B (see Section 3.2.2).

$\mathrm{g}$ "Red" lobe of IRAS 4A is in northeast, and includes blue channels $2.73-5.91 \mathrm{~km} \mathrm{~s}^{-1}$, in addition to red channels.

${ }^{\mathrm{h}}$ Area and volume are calculated only for the emission features marked in Figure 9. PA, $a$ and $b$ for SK 1 are based on the assumption that these features extend from the source SK 1 to form the outflow lobes. 
Table 5

Outflows and their Sources

\begin{tabular}{|c|c|c|c|c|c|c|c|c|c|c|c|c|c|}
\hline \multirow[b]{2}{*}{ Name } & \multicolumn{4}{|c|}{ Driving Source $^{\mathrm{a}}$} & \multicolumn{3}{|c|}{ Blue Lobe } & \multicolumn{3}{|c|}{ Red Lobe } & \multicolumn{3}{|c|}{ Sum } \\
\hline & $\begin{array}{l}\text { YSO } \\
\text { Class }\end{array}$ & $\alpha$ & $\begin{array}{l}T_{\mathrm{Bol}} \\
(\mathrm{K})\end{array}$ & $\begin{array}{l}L_{\text {Bol }} \\
\left(L_{\odot}\right)\end{array}$ & $\begin{array}{l}\text { Mass } \\
\left(M_{\odot}\right)\end{array}$ & $\begin{array}{c}\text { Momentum } \\
\left(M_{\odot} \mathrm{km} \mathrm{s}^{-1}\right)\end{array}$ & $\begin{array}{c}\text { Energy } \\
\left(10^{43} \mathrm{erg}\right)\end{array}$ & $\begin{array}{l}\text { Mass } \\
\left(M_{\odot}\right)\end{array}$ & $\begin{array}{c}\text { Momentum } \\
\left(M_{\odot} \mathrm{km} \mathrm{s}^{-1}\right)\end{array}$ & $\begin{array}{c}\text { Energy } \\
\left(10^{43} \mathrm{erg}\right)\end{array}$ & $\begin{array}{l}\text { Mass } \\
\left(M_{\odot}\right)\end{array}$ & $\begin{array}{c}\text { Momentum } \\
\left(M_{\odot} \mathrm{km} \mathrm{s}^{-1}\right)\end{array}$ & $\begin{array}{c}\text { Energy } \\
\left(10^{43} \mathrm{erg}\right)\end{array}$ \\
\hline SVS $13 A^{b}$ & I & 1.02 & 250 & 59 & 1.0 & 2.7 & 9.8 & 0.7 & 2.2 & 7.8 & 1.7 & 4.6 & 17.6 \\
\hline SVS 13C & 0 & $\cdots$ & $36^{\mathrm{c}}$ & $4.9^{c}$ & 0.8 & 2.5 & 9.7 & 1.1 & 3.3 & 12.1 & 1.8 & 5.0 & 21.8 \\
\hline IRAS 2A-WE & 0 & 2.71 & 57 & 76 & 0.1 & 0.3 & 1.4 & 0.1 & 0.2 & 0.8 & 0.2 & 0.6 & 2.1 \\
\hline IRAS $2 \mathrm{~A}-\mathrm{SN}^{\mathrm{d}}$ & 0 & 2.71 & 57 & 76 & 0.5 & 1.8 & 8.1 & 0.4 & 1.3 & 4.7 & 0.9 & 3.7 & 12.8 \\
\hline $\operatorname{IRAS} 4 \mathrm{~A}^{\mathrm{e}}$ & 0 & 2.55 & 43 & 5.8 & 0.2 & 0.6 & 2.3 & 0.1 & 0.4 & 1.4 & 0.3 & 1.7 & 3.7 \\
\hline IRAS 4B & 0 & 0.87 & 55 & 1.1 & 0.01 & 0.04 & 0.2 & 0.01 & 0.03 & 0.1 & 0.02 & 0.1 & 0.3 \\
\hline SK 14 & 0 & 1.41 & 59 & 0.2 & 0.1 & 0.4 & 1.3 & 0.1 & 0.1 & 0.5 & 0.2 & 0.7 & 1.8 \\
\hline SK 1 & 0 & 2.07 & 32 & 0.7 & 0.1 & 0.5 & 2.5 & 0.1 & 0.2 & 0.6 & 0.2 & 1.0 & 3.1 \\
\hline $\mathrm{C} 1$ & $\cdots$ & $\cdots$ & $\cdots$ & $\cdots$ & $\cdots$ & $\cdots$ & $\cdots$ & 0.1 & 0.2 & 0.7 & 0.1 & 0.2 & 0.7 \\
\hline $\mathrm{C} 2$ & $\cdots$ & $\ldots$ & $\cdots$ & $\cdots$ & 0.2 & 0.7 & 3.3 & $\ldots$ & $\cdots$ & $\ldots$ & 0.2 & 0.7 & 3.3 \\
\hline $\mathrm{C} 3$ & $\cdots$ & $\cdots$ & $\cdots$ & $\cdots$ & $\cdots$ & $\cdots$ & $\cdots$ & 0.3 & 0.8 & 2.8 & 0.3 & 0.8 & 2.8 \\
\hline $\mathrm{C} 4$ & $\cdots$ & $\cdots$ & $\cdots$ & $\cdots$ & $\cdots$ & $\cdots$ & $\cdots$ & 0.2 & 0.5 & 1.6 & 0.2 & 0.5 & 1.6 \\
\hline Sum & & & & & 3.0 & 9.5 & 38.4 & 3.0 & 9.3 & 33.3 & 6.0 & 18.8 & 71.7 \\
\hline
\end{tabular}

Notes.

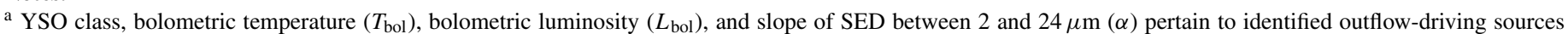
from Evans et al. (2009), except where marked otherwise.

b "Blue" lobe of SVS 13A includes red channels $9.08-11.30 \mathrm{~km} \mathrm{~s}^{-1}$, in addition to blue channels.

${ }^{c} T_{\text {bol }}$ and $L_{\text {bol }}$ from Chen et al. (2009)

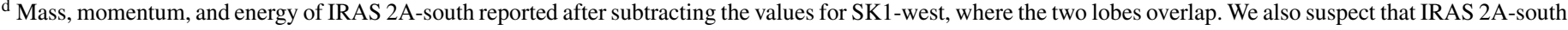

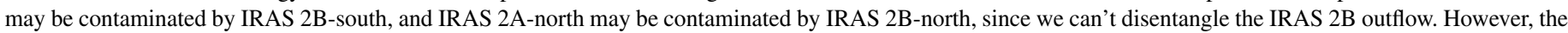
majority of emission appears to be associated with IRAS 2A. See Section 3.2.2 for more discussion.

e "Red" lobe of IRAS 4A includes blue channels $2.73-5.91 \mathrm{~km} \mathrm{~s}^{-1}$, in addition to red channels.

clear orthogonal flows, with IRAS 2A south-north appearing to overlap in the north with the SVS 13A outflow and in the south it may extend beyond the region that we have mapped here.

It has been more recently proposed that IRAS $2 \mathrm{~A}$ may in fact be a binary (Chen et al. 2013), and we therefore suggest that the nearly perpendicular outflows that we resolve are being driven by the two components of this binary. In similar cases where other quadrupolar outflows have been found, subsequent observations with sufficient resolution have revealed that the central protostellar source is a binary, with each component driving a bipolar outflow (e.g., L723, Launhardt 2004; HH111, Reipurth et al. 1999; CG30, Chen et al. 2008).

Characteristics of the IRAS 2A outflows are given in Tables 4 and 5 . The three velocity regimes (low-, medium-, and highvelocity for both blueshifted and redshifted outflowing gas) are shown in Figure 6, and we find that for IRAS 2A west-east the high-velocity outflowing gas is more collimated than the lowvelocity gas. Specifically, we find that in IRAS 2 A west-east, the high-velocity blueshifted and redshifted outflow lobes at their widest points have widths of $\sim 16^{\prime \prime}$, whereas the low-velocity outflow lobes have widths of $\sim 22^{\prime \prime}(\sim 0.018-0.025 \mathrm{pc})$. Gueth \& Guilloteau (1999) see similar morphology in the source $\mathrm{HH}$ 211, where high-velocity CO emission shows a collimated jetlike structure and low-velocity emission traces outflow cavities. The morphology of this outflow is in agreement with the jetdriven outflow paradigm, with low-velocity $\mathrm{CO}$ cavities present in the wake of shocks imparted by the high-velocity protostellar jet. The relatively small overlap of blue and red lobes near the driving source IRAS $2 \mathrm{~A}$ suggests that we are observing IRAS 2A west-east approximately in the plane of the sky with low inclination angle.

The IRAS 2A south-north outflow, approximately perpendicular to the IRAS 2A west-east outflow, is composed of a blueshifted (IRAS 2A-south) lobe extending $188^{\prime \prime}(0.21 \mathrm{pc})$ south of IRAS $2 \mathrm{~A}$ and a redshifted (IRAS 2A-north) lobe ex- tending $133^{\prime \prime}(0.15 \mathrm{pc})$ north. The blue IRAS $2 \mathrm{~A}$-south has an opening angle of $\sim 56^{\circ}$, seen most clearly in low- and midvelocity outflow emission near IRAS $2 \mathrm{~A}$. At $\sim 50^{\prime \prime}(\sim 0.06 \mathrm{pc})$ south of IRAS $2 \mathrm{~A}$, the gas shows nearly parallel cavity walls separated by $\sim 70^{\prime \prime}(\sim 0.08 \mathrm{pc})$. Similar morphology has been seen for the RNO 91 outflow mapped by Lee et al. (2002) and Lee \& Ho (2005). In some channels and pixels, emission of IRAS 2A-south may be confused with a southern, comparably widespread outflow lobe driven by IRAS $2 B$ to the east. In particular, in the low- and mid-velocity channels the eastern cavity wall of IRAS 2A-south may be seen projected between the eastern and western cavity walls of IRAS $2 \mathrm{~B}$, allowing us to make a tentative distinction of the morphologies (see proposed morphology outlined in Figure 6). Another confusion comes from SK 1 (see Section 3.3.4), which overlaps with IRAS 2A-south in the southern region of our map (see Figures 6 and 9). Since in this overlap region most emission appears morphologically related to SK 1 (i.e., position angle, elongation approximately west-east rather than south-north) we associate the emission with SK 1 and subtract the mass, momentum, energy, and area of SK 1 from that of IRAS 2A-south to determine the values reported in Tables 4 and 5.

Close to the source, IRAS 2A-north also shows a wide opening angle $\left(\sim 93^{\circ}\right)$, particularly at low velocities (see left panel of Figure 6). Farther from the source, at $\sim 60^{\prime \prime}(\sim 0.07 \mathrm{pc})$ north of the driving source, IRAS $2 \mathrm{~A}$-north has cavity walls as wide as $90^{\prime \prime}(0.10 \mathrm{pc})$. The extent to which we can identify outflow emission associated with IRAS $2 \mathrm{~A}$ to the north is limited by "contamination" from redshifted outflow emission associated with SVS 13. Knee \& Sandell (2000) suggested that the IRAS 2A south-north outflow, which they call IRAS 2 NNE-SSW, may have blueshifted emission extending at least $4^{\prime}(0.27 \mathrm{pc})$ to the south, and therefore it is possible that in spite of confusion with surrounding, strong outflows, the redshifted lobe may extend an equal distance to the north. Further, Knee 


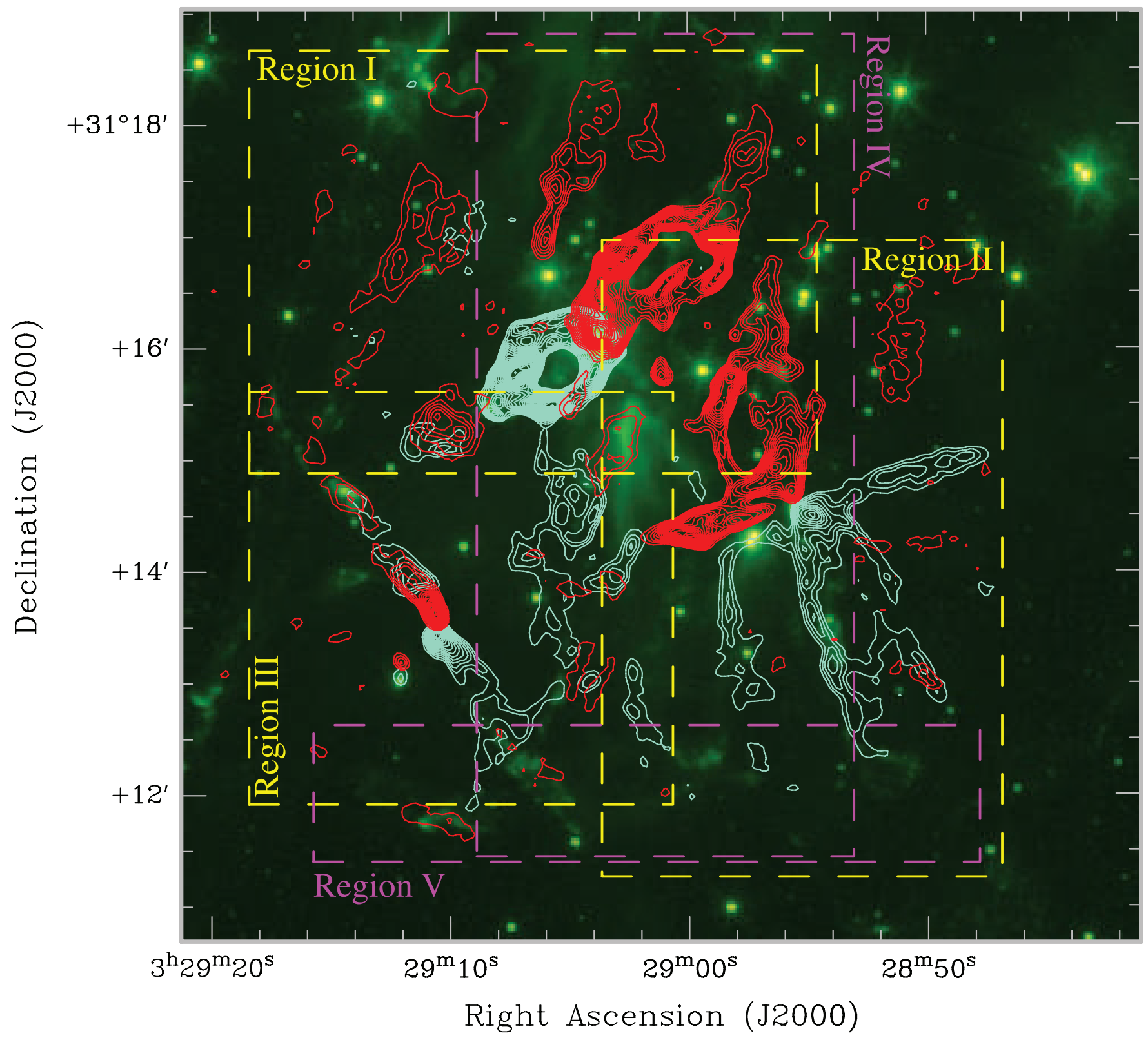

Figure 4. ${ }^{12} \mathrm{CO}$ emission in the mapped region of NGC 1333 (contours), overlaid on a map of IRAC $4.5 \mu \mathrm{m}$ emission (Gutermuth et al. 2008). Contours show integrated intensity of blueshifted and redshifted emission in channels with velocity $V_{\mathrm{LSR}}=-2.3-6.2 \mathrm{~km} \mathrm{~s}^{-1}$ (blue) and $V_{\mathrm{LSR}}=10.0-17.3 \mathrm{~km} \mathrm{~s}-1$ (red). Contours begin with $2 \sigma$ and increment by $2 \sigma$, where $\sigma$ is the rms of the respective moment map. We detect outflow emission greater than $3 \sigma$ spanning a region of approximately $7^{\prime} \times 7^{\prime}$ in size (see Section 2.1). Dashed boxes indicate the regions we show with more detail in Figures 5-9 and are described in more detail in Sections 3.2 and 3.3. In the following figures, we show integrated intensity maps for low-, mid-, and high-velocity CO emission in Regions I-III (yellow dashed boxes), and for Regions IV-V (magenta dashed boxes) we show just the blueshifted and redshifted velocity channels where most outflow emission is found.

(A color version of this figure is available in the online journal.)

\& Sandell (2000) claim that IRAS 2 NNE extends north beyond the red lobe of SVS 13, and they calculate more mass associated with IRAS 2 NNE to the north of SVS 13 than to the south.

It is apparent in Figure 6 that the morphology of IRAS 2A south-north is distinct from that of IRAS 2A west-east. IRAS 2A south-north spans approximately five times more area on the plane of the sky than IRAS 2A west-east, and we measure about seven times more mass and energy associated with IRAS 2A south-north (see Tables 4 and 5). Since IRAS 2A south-north is less collimated than IRAS 2A west-east, and the outflow lobes of IRAS 2A south-north also extend farther so that they are confused with other outflows in our map, several of the characteristics of IRAS 2A south-north are more difficult to determine.

The third outflow in the region, which we tentatively associate with the source IRAS 2B mentioned above, has a clumpy morphology that is further confused by the strong nearby IRAS 2A outflows, and some or all of this clumpy structure may in fact be driven by IRAS 2A, or the candidate outflow SVS 13Csouth, described in Section 3.3.1. Knee \& Sandell (2000) detect faint, tentative outflow emission extending about $1^{\prime}$ south of IRAS 2B, while the map of Engargiola \& Plambeck (1999) shows blueshifted emission 2.5 south and redshifted emission 1.5 north that may be associated with IRAS 2B. 

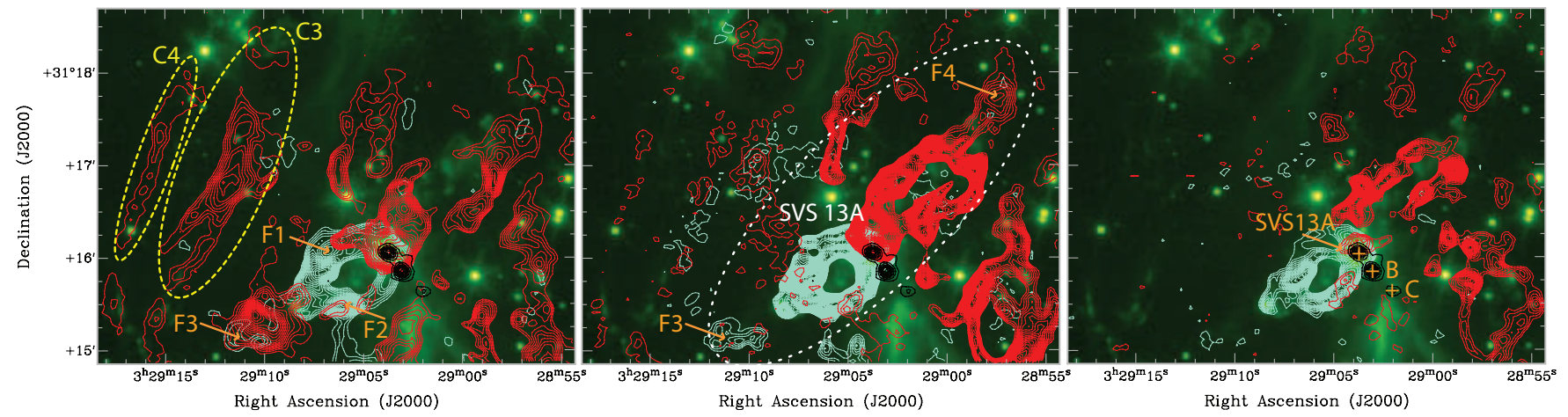

Figure 5. Region I low-, mid-, and high-velocity outflows (left, middle, and right panels, respectively). Blueshifted and redshifted CO outflows are shown with blue and red contours, respectively, continuum emission is shown with black contours, and background is IRAC $4.5 \mu$ m emission (Gutermuth et al. 2008). Continuum sources SVS 13A, SVS 13B, and SVS 13C are marked with plus signs and labeled only in the right panel. Outflows in this region are approximately outlined with ellipses, to guide the eye. The SVS 13 outflow lobes (white dotted ellipse) have "donut" morphologies and are driven by SVS 13A. F1 and F2 mark the locations of the strongest clumpy emission features in our map, and F3 and F4 mark two distinct "blobs" of emission at the tips of both lobes. Located northeast of SVS 13 are the candidate outflows C 3 and C4 (yellow dashed ellipses), seen predominantly in low-velocity redshifted emission. We group channels as described at the beginning of Section 3.2. Contours begin with $3 \sigma$ and increment by $3 \sigma$ for each $\mathrm{CO}$ integrated intensity map, and begin with $4 \sigma$ and increment by $4 \sigma$ for continuum.

(A color version of this figure is available in the online journal.)
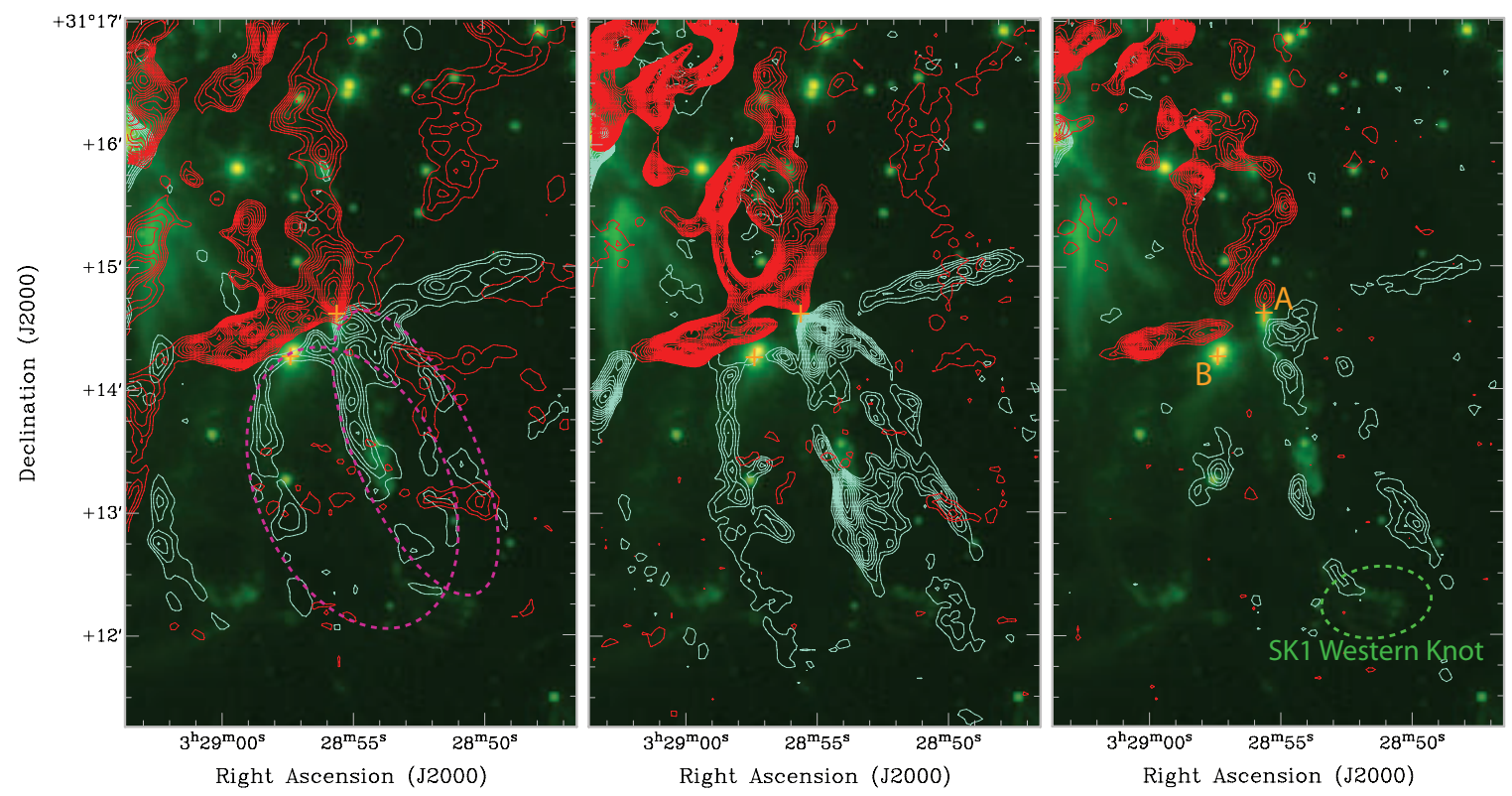

Figure 6. Region II low-, mid-, and high-velocity outflows (left, middle, and right panels, respectively). Blueshifted and redshifted CO outflows are shown with blue and red contours, respectively, and background is IRAC $4.5 \mu \mathrm{m}$ emission (Gutermuth et al. 2008). Continuum sources IRAS 2A and IRAS 2B are marked with plus signs and labeled only in the right panel. Magenta ellipses show the proposed morphology for the southern lobes of the outflows driven by sources IRAS $2 \mathrm{~B}$ and IRAS 2A (i.e., the lobes IRAS 2B-south and IRAS 2A-south). Green dashed ellipse marks the region where emission overlaps with the SK1 western knot (see Figure 9). We group channels as described at the beginning of Section 3.2. Contours begin with $3 \sigma$ and increment by $3 \sigma$ for each CO integrated intensity map.

(A color version of this figure is available in the online journal.)

Figure 6 shows our suggested outflow morphology, with similar wide angle outflows emanating south from IRAS 2B (hereafter IRAS 2B-south) as in the case of IRAS 2A-south. The opening angle of IRAS $2 \mathrm{~B}$-south is $\sim 60^{\circ}$, seen most clearly in low-velocity channels with $v_{\text {out }}<7 \mathrm{~km} \mathrm{~s}^{-1}$, and beyond $\sim 50^{\prime \prime}$ $(\sim 0.06 \mathrm{pc})$ south of IRAS $2 \mathrm{~B}$ the cavity walls are nearly parallel, similar to the morphology of IRAS 2A-south. The proposed northern lobe, IRAS 2B-north, is confused with IRAS 2A-north and the outflow from SVS 13, but particularly in low-velocity channels, emission close to the IRAS 2B continuum source suggests that this source is in fact driving an outflow to the north. Because we cannot disentangle the outflow from IRAS $2 B$ and the outflows from IRAS $2 \mathrm{~A}$ and SVS 13C, we do not include a mass estimate for the IRAS 2B outflow. We acknowledge that the mass estimates of the IRAS $2 \mathrm{~A}$ and SVS $13 \mathrm{C}$ outflows may be over-estimated due to some contribution from IRAS 2B, however our total outflow mass estimate for the region includes the emission pertaining to the IRAS 2B, IRAS 2A, and SVS 13C outflows.

\subsubsection{IRAS 4 Outflows}

Among the Class 0 continuum sources in the IRAS 4 region, described in Section 3.1.3, the two brightest continuum sources IRAS 4A and IRAS 4B are driving outflows, while IRAS 4C and IRAS 4D appear to have no associated outflow emission. In a detailed molecular line survey, Blake et al. (1995) associate outflow emission with IRAS 4A and IRAS 4B, with IRAS 4A driving a highly collimated jet-like bipolar outflow, and IRAS 4B driving a more compact outflow evidenced by high-velocity wings in the spectrum at the position of the source. Curtis 

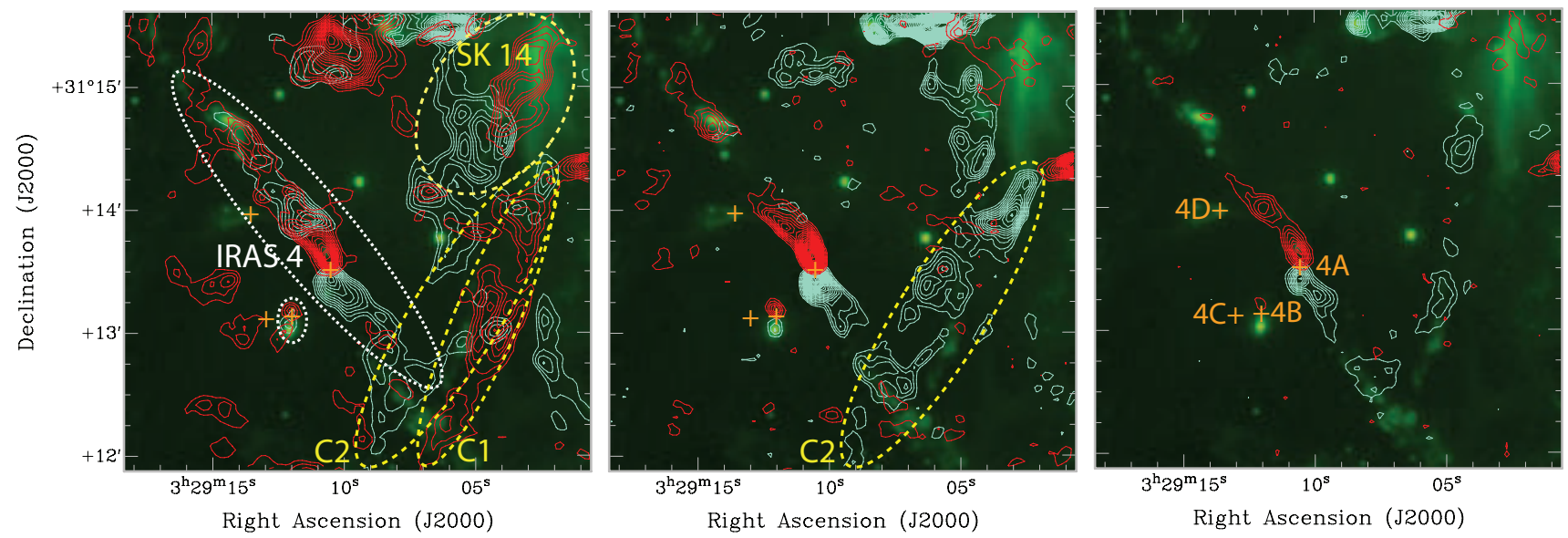

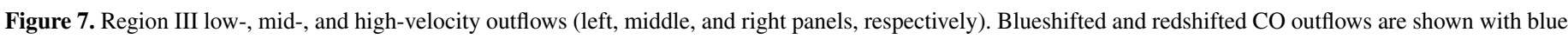

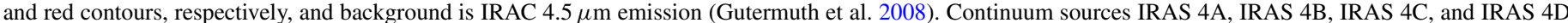

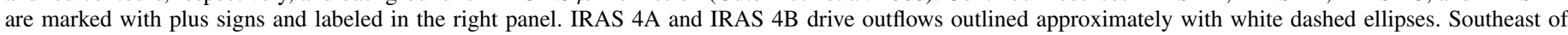

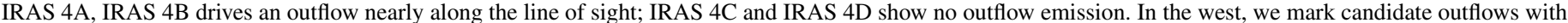

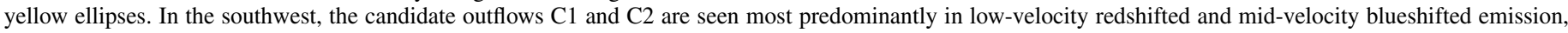

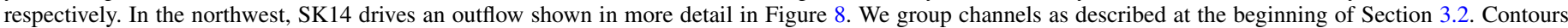
begin with $3 \sigma$ and increment by $3 \sigma$ for each $\mathrm{CO}$ integrated intensity map.

(A color version of this figure is available in the online journal.)

et al. (2010b, see their Figure A3) detects CO outflow emission associated with both IRAS 4A and IRAS 4B, with the red outflow lobes associated with the two sources resolved at both high- and low-velocities, but the blue lobe of IRAS 4B does not appear in their low-velocity map and appears unresolved in their high-velocity map. We detect outflow emission associated with IRAS 4A and IRAS 4B to have distinct morphologies, as suggested by the previously mentioned observations, and in our maps the outflows associated with the two sources are resolved at both low- and high-velocities.

As seen in our map shown in Figure 7, IRAS 4A drives a very collimated outflow, with the blueshifted lobe toward the south-southwest (hereafter IRAS 4A-south) and the (mostly) redshifted lobe toward the north-northeast (hereafter IRAS 4Anorth). The maximum extent of the blue and red lobes that we measure are $104^{\prime \prime}(0.12 \mathrm{pc})$ and $125^{\prime \prime}(0.14 \mathrm{pc})$, respectively. The extent is large enough that both south and north lobes appear to be confused with other outflow emission in the southwest and the north, making it difficult to determine their full extent. Further, the north lobe extends to the edge of our map in the east. We note that we detect outflow emission associated with IRAS 4A at all velocity channels with $\left|v_{\text {out }}\right|>2 \mathrm{~km} \mathrm{~s}^{-1}$, even at the outermost channels in our band. In fact, Curtis et al. (2010b) show that for this source, there is faint outflow emission out to $\left|v_{\text {out }}\right|=20 \mathrm{~km} \mathrm{~s}^{-1}$ (see the spectra in their Figure B1), and hence our estimates for the mass, momentum, and kinetic energy of this outflow should be considered only as lower limits.

Within $\sim 20^{\prime \prime}$ of IRAS $4 \mathrm{~A}$, and most notably in the highand mid-velocity maps, outflow lobes appear to extend nearly north-south, but beyond this distance we find different position angles with decreasing velocities. Beyond $20^{\prime \prime}$ we measure position angles of $57^{\circ} \pm 1^{\circ}, 51^{\circ} \pm 2^{\circ}$, and $43^{\circ} \pm 3^{\circ}$ for the low-, mid-, and high-velocity outflows, respectively. Fitting an ellipse to the entire outflow and over all velocities, we find a mean position angle of $\sim 40^{\circ}$. The blueshifted and redshifted lobes intersect but only barely overlap, so the outflows appear to be oriented nearly in the plane of the sky. We calculate a total outflow mass of $0.3 M_{\odot}$ associated with IRAS 4A. However, we note in Section 3.3.4 that the candidate outflow lobe C2 likely crosses IRAS 4A-south, and we therefore consider that the mass of IRAS 4A-south may be slightly over-estimated.

Choi (2001) observed HCN as a tracer of the outflows in the IRAS 4 region, and they found compact knots associated with IRAS 4A that follow a "wiggle" pattern rather than forming a straight trajectory from the driving source. Later, based on an $\mathrm{SiO}$ (1-0) map, Choi (2005) measured a change of position angle of the northern collimated lobe, which they called a bend, of about $34^{\circ}$. The location of this bend (about $25^{\prime \prime}$ north of IRAS 4A) is in agreement with the morphology we see in our map and in the CO (2-1) map by Girart et al. (1999). The CO (2-1) reveals a change in PA of about $45^{\circ}$, which is closer to the mean PA that we measure.

Several conclusions can be drawn from the collimated yet "wiggly/bent" outflow from IRAS 4A. Choi (2001) suggest several mechanisms for directional and intensity variability, although some of the suggested mechanisms only account for one or the other type of variability, and a combination of mechanisms is likely necessary. Precession and episodic ejection together adequately account for both the "wiggles/bends" (directional variability) and knots (intensity variability) seen in IRAS 4A. We observe that position angles for both the blueshifted and redshifted lobes are consistent, and in particular that gas farther from the source has the largest position angle, so the outflow axis appears to be precessing in the clockwise direction. The precession may be explained by the fact that IRAS 4A is a binary (see Section 3.1.3). Terquem et al. (1999) suggest that the position axis of an outflow may precess due to the precession of the source, which itself could be caused by the tidal interaction between the circumstellar disk of the outflow source and one (or many) stellar companion(s).

It can also be seen in Figure 7 that the northern lobe of the IRAS 4A outflow consists of blueshifted and redshifted outflow emission (specifically at low-velocities), whereas the southern lobe is almost entirely blueshifted. In particular, in IRAS 4A-north we detect high-velocity redshifted emission spatially coincident with lower-velocity blueshifted emission (in channels with $v_{\mathrm{LSR}}=3-17 \mathrm{~km} \mathrm{~s}^{-1}$ ). The blueshifted emission has a bow-shaped morphology, and it is coincident with the position of the bend in the northern lobe. Since the 
blueshifted and redshifted emission follow a continuous outflow trajectory to the north of IRAS 4A, we suggest that both components comprise the same northern outflow lobe, and the spatial coincidence of the redshifted and blueshifted outflow emission is consistent with the scenario of precession about an axis approximately parallel to the plane of the sky.

The observed IRAS 4B outflow kinematics and morphology differ drastically from IRAS 4A. Although it is also driven by a Class 0 source, IRAS 4B shows weaker outflow emission than IRAS $4 \mathrm{~A}$, and a more substantial fraction of the emission is low- and mid-velocity. In contrast to IRAS 4A, the IRAS 4B outflow appears oriented along the line of sight and it is very compact as seen in Figure 7. Possible projection scenarios for the IRAS 4B outflow relative to that from IRAS 4A are presented by Yildiz et al. (2012) based on multi-transition observations of CO and several of its isotopologues, with models assuming that the intrinsic lengths of the outflows are similar. We calculate a mass of $0.02 M_{\odot}$ for IRAS $4 \mathrm{~B}$, with each lobe only extending about $10^{\prime \prime}$ on the plane of the sky, but spanning about $v_{\text {cloud }} \pm 5 \mathrm{~km} \mathrm{~s}^{-1}$ in radial velocity. This extent is in agreement with those reported by Y1ldiz et al. (2012) based on CO (3-2) and CO (6-5) observations made with JCMT and APEX, although in those observations the outflow lobes were reported as unresolved.

As stated previously, we detect continuum emission attributed to both binary components IRAS 4B and IRAS 4C, but no outflow emission associated with IRAS 4C was detected. Similarly we do not detect outflow emission associated with the weaker continuum source IRAS 4D to the northeast.

\subsection{Outflow Candidates}

Here we present several outflow candidates based on ${ }^{12} \mathrm{CO}$ emission morphology that were previously unidentified or confused with stronger surrounding outflows. We associate candidate $\mathrm{CO}$ outflow emission with the protostellar sources SVS 13C, SK 14, and SK 1. The candidate outflows that we cannot associate with a driving source are labeled C1, C2, C3, and $\mathrm{C} 4$.

\subsubsection{SVS $13 C$}

In Section 3.1.1, we described three continuum sources detected in the SVS 13 region. Of these, SVS 13A is the continuum source associated with the IR source SVS 13 driving the bipolar HH 7-11 outflow, which we described in Section 3.2.1. In addition to this outflow, we also detect molecular outflow emission extending south-north on the plane of the sky that appears to be associated with the southernmost source in the group, SVS 13C (Region IV in Figure 4; see also Figure 8). Outflow emission south of SVS 13 has previously been observed (e.g., Knee \& Sandell 2000; Davis et al. 2008; Curtis et al. 2010b), but the driving source has not been conclusively or consistently identified. Knee \& Sandell (2000) associate this emission with an outflow driven by SVS 13B, but they also acknowledge that a dust shell found south of SVS 13 (Lefloch et al. 1998; Sandell $\&$ Knee 2001) may have been created by SVS 13B or SVS 13 C (they use the name $\mathrm{H}_{2} \mathrm{O}$ (B) for this source).

Here we provide more evidence that this outflow is driven by SVS 13C, and we propose a northern outflow lobe counterpart. South and north of SVS $13 \mathrm{C}$ we detect CO emission up to $\left|v_{\text {out }}\right|=9 \mathrm{~km} \mathrm{~s}^{-1}$ along an axis with position angle $8^{\circ}$, pertaining to blue and red lobes extending $\sim 212^{\prime \prime}(0.24 \mathrm{pc})$ south and $\sim 180^{\prime \prime}(0.21 \mathrm{pc})$ north, respectively (hereafter SVS 13C-south and SVS 13C-north).

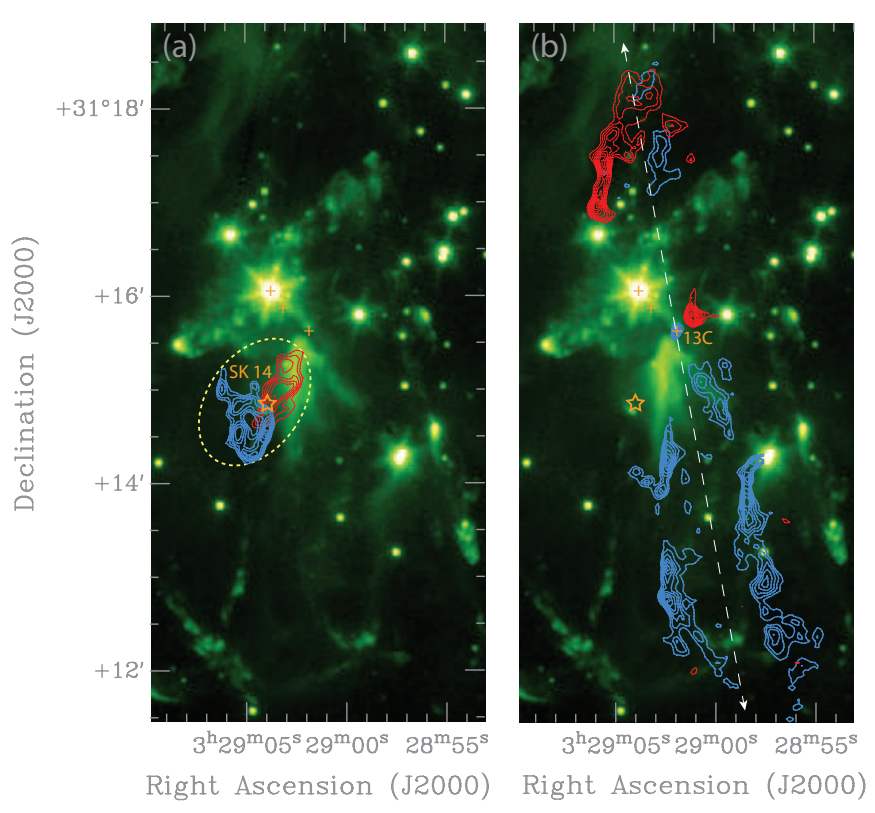

Figure 8. Candidate outflows associated with SK 14 (panel (a)) and SVS 13C (panel (b)), corresponding to Region IV in Figure 4. (a) Contours are integrated intensity for channels with $v_{\mathrm{LSR}}=-1-6 \mathrm{~km} \mathrm{~s}^{-1}$ (blue) and $v_{\mathrm{LSR}}=$ 9-11 $\mathrm{km} \mathrm{s}^{-1}$ (red), beginning with $3 \sigma$ and incrementing by $\sigma$, where $\sigma$ is the rms of the respective integrated intensity maps. (b) Contours are integrated intensity for channels with $v_{\mathrm{LSR}}=4-6 \mathrm{~km} \mathrm{~s}^{-1}$ (blue) and $v_{\mathrm{LSR}}=12-14 \mathrm{~km} \mathrm{~s}^{-1}$ (red), beginning with $3 \sigma$ and incrementing by $3 \sigma$. For clarity in each panel of this figure, we include only contours pertaining to the outflows SK 14 and SVS 13C, and we erased the contours of outflow emission from other nearby sources (e.g., SVS 13A). Crosses mark the continuum sources in the SVS 13 region, and the star symbol marks SK 14, for which we did not detect continuum emission. The dashed white line in (b) marks an axis for the SVS 13C north-south outflow with position angle $8^{\circ}$, fit by eye. Arrows suggest that the outflow lobes may extend beyond the edge of our map. Background image is IRAC $4.5 \mu \mathrm{m}$ emission.

(A color version of this figure is available in the online journal.)

Particularly in SVS 13C-south, CO emission approximately follows the nebula seen in $4.5 \mu \mathrm{m}$ emission, shown in Figure 8. In the far south $\mathrm{CO}$ outflow emission is coincident with $4.5 \mu \mathrm{m}$ emission that has a bow-shaped morphology and which may be a shock from the SVS 13C-south flow. Proper motions for this structure are up to $100 \mathrm{~km} \mathrm{~s}^{-1}$ in the southern direction (Raga et al. 2013), and such high velocity and large spatial extent on the plane of the sky suggest a low inclination of this outflow lobe with respect to the plane of the sky. Some of the emission detected in the southwestern component of SVS 13C-south may also be contributed by the IRAS 2B outflow (see Figure 6), but as can be seen in Figure 8 the emission is consistent spatially with an outflow lobe driven by SVS 13C.

To the north of SVS 13C, we see strong emission comprising the red outflow lobe SVS 13C-north which counters the blue outflow lobe SVS 13C-south, but which is also confused with the northwest red SVS 13A outflow lobe described in Section 3.2.1, or possibly the northern outflow lobe driven by IRAS 2 (Knee \& Sandell 2000). Another possibility is that this emission may be associated with a source at or beyond the northern edge of our map, further reason to extend the map to the north in order to more conclusively identify the counterpart lobes. However, based on our map, we suggest that SVS 13C-south and SVS 13Cnorth are the blue and red lobes of the molecular outflow associated with SVS 13C. For clarity, in Figure 8 we show the SVS 13 region with contours only pertaining to emission which we associate with SVS 13C but not the SVS 13A SE-NW outflow. Since both lobes of the SVS 13C outflow shown in 


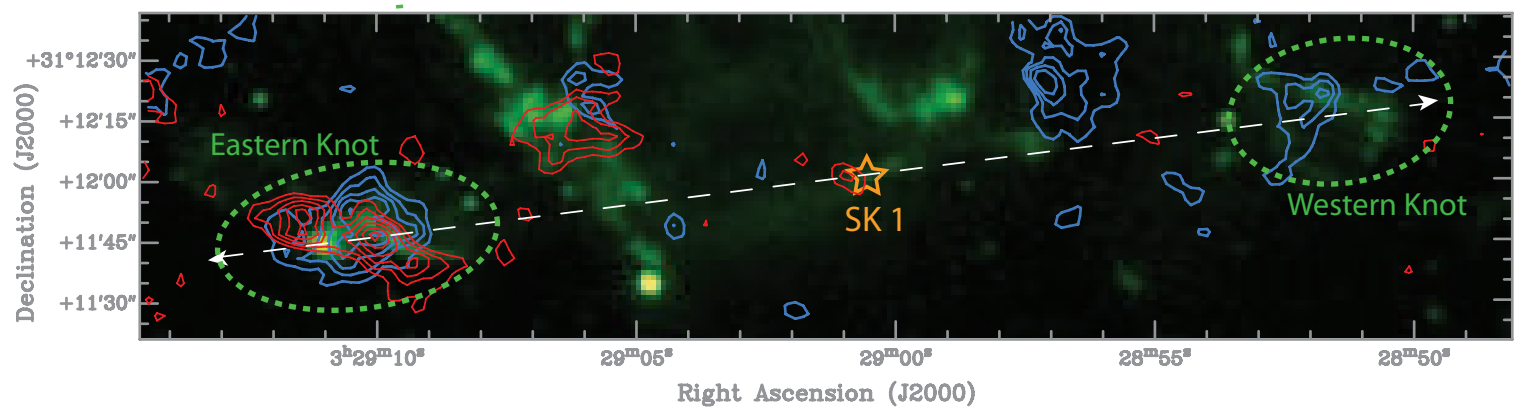

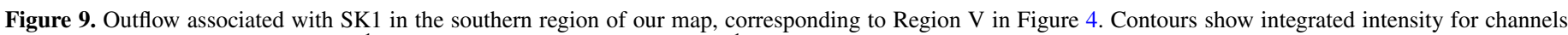

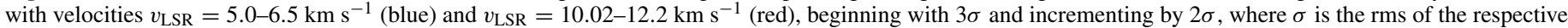

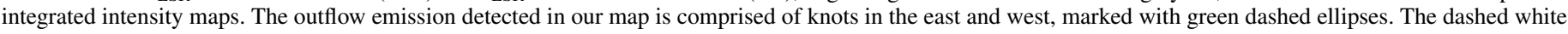

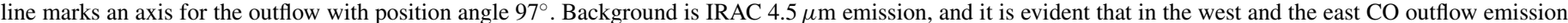
is coincident with bow-shocks.

(A color version of this figure is available in the online journal.)

Figure 8 extend out to the edge of our map, it is very likely that these lobes extend even farther than what we map here.

Near the position of the SVS 13C continuum source are two concentrated emission blobs in blueshifted and redshifted channels up to $\left|v_{\text {out }}\right|=5 \mathrm{~km} \mathrm{~s}^{-1}$. The blueshifted blob is coincident with the position of SVS $13 \mathrm{C}$, and the redshifted blob extends northwest only $\sim 10^{\prime \prime}-20^{\prime \prime}$ (projected on the plane of the sky) from SVS 13C, reminiscent of the IRAS 4B outflow. One possible explanation is that these two blobs represent the lobes of an outflow that is oriented nearly along the line of sight (similar to the IRAS 4B outflow) and perpendicular to the main north-south outflow driven by SVS 13C. If this is the case, then SVS $13 C$ is likely a binary source driving two bipolar outflows. Alternatively, the blobs may be part of the wall of the SVS 13Cnorth outflow lobe. Further observations are needed to explain this emission structure.

\subsubsection{SK 14 Candidate Outflow}

In the region southeast of SVS 13 and northwest of IRAS 4, we detect a bipolar outflow oriented southeast-northwest seen particularly in low-velocity channels with $\left|v_{\text {out }}\right|=2-4 \mathrm{~km} \mathrm{~s}^{-1}$, but also with blueshifted emission at higher velocities (see Figure 8, also Figure 7). The outflow lobes intersect at the location of the Class 0 source SK 14 detected by Sandell \& Knee (2001), which in addition to sub-mm detections (Sandell \& Knee 2001; Hatchell et al. 2005) also has counterparts at radio (Rodríguez et al. 1999), millimeter (Enoch et al. 2006) and IR (Gutermuth et al. 2008; Evans et al. 2009) wavelengths. As indicated in Table 2, this is a Class 0 source from SED-based indicators (e.g., Hatchell et al. 2007b). In previous outflow studies, such as that of Hatchell et al. (2007a, see their Table 1) who observed CO (3-2) with JCMT, the outflow associated with this source is suggested, but has been confused by nearby stronger outflow emission, particularly emission related to SVS 13. The spectral and spatial resolution of our observations allow us for the first time to distinguish the velocity structure and morphology of the outflow lobes. This also has implications for distinguishing the candidate outflow lobes $\mathrm{C} 1$ and $\mathrm{C} 2$, described in Section 3.3.4.

We detect emission associated with SK 14 in blue channels with velocities up to $\left|v_{\text {out }}\right|=9 \mathrm{~km} \mathrm{~s}^{-1}$, whereas emission in the red channels is only seen up to $\left|v_{\text {out }}\right|=3 \mathrm{~km} \mathrm{~s}^{-1}$. At low velocities, the blue outflow has a donut-shaped morphology and "clumpy" emission similar to that of SVS 13, although the structure is smaller and the integrated emission is not as strong.
The redshifted outflow emission remains narrower and weaker than the blue emission, and the northern extent of the outflow lobe may be confused with SVS 13 and other strong outflows to the north and east. We measure a position angle of $145^{\circ}$, and blueshifted and redshifted outflow lobe extents of $71^{\prime \prime}(0.08 \mathrm{pc})$ and $58^{\prime \prime}(0.07 \mathrm{pc})$, respectively.

\subsubsection{SK 1 Candidate Outflow}

The source SK 1 (Sandell \& Knee 2001), also identified as Bolo 41 (Enoch et al. 2006) and HRF 65 (Hatchell et al. 2007b; Hatchell \& Dunham 2009; Curtis et al. 2010b), lies at the southern edge of our map (Region V of Figure 4, see also Figure 9), and has been previously classified as a Class 0 object (see Table 2). Curtis et al. (2010b) show that the source drives a bipolar outflow extending east-west nearly $10^{\prime}$, with the red lobe to the east and the blue lobe to the west, both extending beyond the edges of our map. The knots that we detect associated with SK 1 have a bow-shape and are coincident with IRAC $4.5 \mu \mathrm{m}$ emission at $\sim 2$.5 $(0.2 \mathrm{pc})$ from the driving source, shown in Figure 9. These features are presumably caused by outflow shocks, characteristic of a young, jet-like outflow. It is very likely that we only detect these distinct blobs of emission, rather than a contiguous outflow morphology, because this source is in the region of our map with the poorest sensitivity and we are only able to detect the brightest blobs of this outflow. We only include these features (see Figure 9) in our calculations pertaining to SK 1 in Tables 4 and 5 .

Based on the fact that the easternmost knot is seen in both blueshifted and redshifted emission, as well as proper motions showing high velocity $\left(\sim 80 \mathrm{~km} \mathrm{~s}^{-1}\right)$ along the plane of the sky (Raga et al. 2013), it is very likely that this outflow has a low inclination angle with respect to the plane of the sky. The emission that we detect is coincident with previous observations, particularly the ${ }^{12} \mathrm{CO}(J=3-2)$ emission observed by Curtis et al. (2010b), and with our higher-resolution maps (see Figure 9) we see that the east-west outflow lobes are about $30^{\prime \prime}$ wide, narrower than previously determined. Some of the emission previously attributed to this outflow may in fact be contributed by several distinct outflows in the region. For example, the blue (west) lobe of SK 1 may be "contaminated" by the blueshifted emission of the southern lobe from SVS 13C or IRAS 2 (discussed above), while in the east we see both red and blue emission that may be associated with other candidate outflows (discussed below). 


\subsubsection{Candidate Outflow Lobes without Identified Sources}

We identify four candidate outflow lobes within the map based on morphology, but for which we cannot identify conclusively their driving sources. In the southeast region of our map (within Region III of Figure 4, shown also in Figure 7) we see a narrow red outflow feature which we name $\mathrm{C} 1$ that extends at least $180^{\prime \prime}(0.2 \mathrm{pc})$. This structure has a position angle of $157^{\circ}$, and is coincident with filamentary emission seen in IRAC $4.5 \mu \mathrm{m}$ emission (Gutermuth et al. 2008) along its northeastern edge. We see emission associated with this feature in red channels with $\left|v_{\text {out }}\right| \lesssim 3 \mathrm{~km} \mathrm{~s}^{-1}$. With a relatively small extent in radial velocity, $\mathrm{C} 1$ is most apparent when inspecting a moment map of only the low-velocity channels (i.e., left panel of Figure 7, rather than Figure 4). Within the extent of our map we do not see any blueshifted emission that could be conclusively identified as the counterlobe of $\mathrm{C} 1$.

Also in Region III and marked in Figure 7, slightly northeast of $\mathrm{C} 1$ we see a candidate outflow lobe $\mathrm{C} 2$ with position angle $148^{\circ}$ and with similar morphology, but with blueshifted velocities $\left|v_{\text {out }}\right| \lesssim 8 \mathrm{~km} \mathrm{~s}^{-1}$. The southwestern edge of $\mathrm{C} 2$ is coincident in projection with the northeastern edge of $\mathrm{C} 1$ and the shocked emission seen in IRAC $4.5 \mu \mathrm{m}$ emission. It is difficult to conclusively associate the shocked emission with either $\mathrm{C} 1$ or $\mathrm{C} 2$, but the position angle of the IRAC filament appears to be more consistent with $\mathrm{C} 1 . \mathrm{H}_{2}$ emission knots in this region have been observed in the near- and mid-IR by Davis et al. (2008) and Maret et al. (2009), respectively, with position angle of about $160^{\circ}-170^{\circ}$, although they were previously associated with SVS 13B, whose tentative outflow is discussed more below.

The blueshifted C2 outflow emission extends at least $122^{\prime \prime}$ $(0.14 \mathrm{pc})$, where the southern tip appears to coincide with the blue southwestern edge of IRAS 4A-south. To the southeast of IRAS 4A-south, there also appears to be emission associated with $\mathrm{C} 2$ and with the same position angle, but since we cannot disentangle $\mathrm{C} 2$ from IRAS 4A-south, and in the region where the two overlap the majority of the emission appears to be associated with IRAS 4A-south, we only include the emission west of IRAS 4A-south in our calculations for C2, presented in Tables 4 and 5.

In previous molecular outflow and $\mathrm{H}_{2}$ observations, an outflow lobe has been tentatively suggested to extend south from SVS 13B, comprising SK 14 and C2 and reaching IRAS 4Asouth. For example, the CO observations of Knee \& Sandell (2000, see their Figures 2 and 3) and Curtis et al. (2010b, see their Figure A3) reveal blueshifted emission in the region between SVS 13 and IRAS 4, but upon inspection of the maps in these two studies it is apparent that the emission does not follow a consistent position angle nor does it have the morphology characteristic of other better-defined outflow lobes in the region. Further, there appears to be a dip in $\mathrm{CO}$ intensity along the previously proposed feature, midway between SVS 13 and IRAS 4. With our higher-resolution maps, having identified the SK 14 outflow (blueshifted and redshifted lobes) and revealing the blueshifted outflow lobe candidate $\mathrm{C} 2$ (in addition to the redshifted lobe $\mathrm{C} 1$ ), we therefore suggest that the observed morphology of the CO emission toward the southeast of SVS 13 and northwest of IRAS 4A-south may instead be explained by several distinct outflow features, for which we cannot conclusively identify the driving source(s).

In Region I in the northeastern corner of our map we detect clumps of redshifted emission, which we name C3 and C4, shown in Figure 5. These structures show elongated outflowlike morphologies, with a position angle of $\sim 160^{\circ}$, and are coincident with extended $4.5 \mu \mathrm{m}$ emission. C3 extends $\sim 200^{\prime \prime}$ $(0.23 \mathrm{pc})$, with a $\sim 50^{\prime \prime}$ blob of emission to the northwest, and a more narrow filamentary structure with nearly parallel walls extending toward the southeast. $\mathrm{C} 4$ is narrower, with length of $144^{\prime \prime}(0.16 \mathrm{pc})$ and width of only $18^{\prime \prime}(0.02 \mathrm{pc})$. We expect the blueshifted outflow lobes associated with $\mathrm{C} 3$ and $\mathrm{C} 4$ to lie beyond the north or east edges of our map. At least one of these outflow features might be associated with the protostar IRAS 7 (see Table 2), which lies at the northern edge of our map and also drives the HH6 jet and associated outflow.

\subsection{Mass, Momentum, and Energy of Outflows}

Here we describe the method for determining mass, momentum, and energy of the outflows based on $\mathrm{CO}$ emission. The outflows described in Sections 3.2 and 3.3 were each fit with an ellipse in order to approximate the outflow morphology and delineate emission associated with an individual outflow. Each ellipse was fit to include the outflow driving source (if identified, with the exception of SK1 explained in footnote of Table 4), as well as the farthest and widest pixels of $\mathrm{CO}$ emission greater than $3 \sigma$, and then each velocity channel was inspected by eye to ensure that all emission greater than $3 \sigma$ that was consistent spatially and spectrally with the individual outflow morphology was included. Parameters of these ellipses, which allowed us to calculate outflow morphology characteristics, are given in Table 4.

Inspecting the channel maps of the combined CARMA+ FCRAO observations, we detect intervening large-scale, smooth $\mathrm{CO}$ emission in channels with velocity between 4 and $10 \mathrm{~km} \mathrm{~s}^{-1}$ likely due to another cloud along the line of sight (see appendix of Arce et al. 2010). This extended emission is not seen in CARMA-only maps because it is filtered out by the interferometer. In the combined interferometer and single dish map, we measured the emission within regions with no outflows, and we found that the intervening emission is approximately uniform in each velocity channel and peaks at $5.9 \mathrm{~km} \mathrm{~s}^{-1}$. For our outflow mass calculations, we subtract this excess emission for each channel where significant extended emission is detected.

We follow the method of Arce \& Goodman (2001) - who followed a modified method of Bally et al. (1999) and Yu et al. (1999) - to estimate the outflow mass, using ${ }^{12} \mathrm{CO}$ and ${ }^{13} \mathrm{CO}$ data to correct for the velocity-dependent opacity of the ${ }^{12} \mathrm{CO}$ line. For each outflow lobe we calculate the average ${ }^{13} \mathrm{CO}$ emission and average ${ }^{12} \mathrm{CO}$ emission for each $0.3 \mathrm{~km} \mathrm{~s}^{-1}$ wide channel, and using the average spectra we determine the ratio of the intensity, $R_{12 / 13}$, within the ellipse used to define the outflow lobe emission. We fit a second-order polynomial to the ratio, constrained to have a minimum value at the velocity of the ambient cloud $\left(v_{\mathrm{LSR}}=8 \mathrm{~km} \mathrm{~s}^{-1}\right)$, and excluding velocity channels closest to the ambient velocity with $v_{\mathrm{LSR}}=$ $6-10 \mathrm{~km} \mathrm{~s}^{-1}$. We truncate the function $R_{12 / 13}$ at 62 , the assumed isotopic ratio (Langer \& Penzias 1993). This parabolic fit is used to extrapolate to the high-velocity wings of the outflow where the ${ }^{13} \mathrm{CO}$ is too weak to be reliably detected (explained below).

We assume that ${ }^{13} \mathrm{CO}$ is optically thin at outflow velocities, and if ${ }^{13} \mathrm{CO}$ emission from a position $\left(x_{i}, y_{i}\right)$ at a velocity $v_{i}$ is greater than or equal to three times the rms noise of the spectrum at that position, then we use the intensity for that velocity, $I_{13}\left(x_{i}, y_{i}, v_{i}\right)$, to estimate the ${ }^{13} \mathrm{CO}$ optical depth and column density (from which we obtain the gas mass). If ${ }^{13} \mathrm{CO}$ emission is less than three times the rms noise, then we use the ${ }^{12} \mathrm{CO}$ emission at that position $\left(x_{i}, y_{i}\right)$ and velocity $v_{i}$ to estimate $I_{13}\left(x_{i}, y_{i}, v_{i}\right)=I_{12}\left(x_{i}, y_{i}, v_{i}\right)\left[R_{12 / 13}\right]^{-1}$. 
We determine optical depth of the ${ }^{13} \mathrm{CO}$ line according to the following equation (Wilson et al. 2009):

$$
\tau_{13}(x, y, v)=-\ln \left[1-\frac{I_{13}(x, y, v)}{T_{0}\left(\left[\exp \left(T_{0} / T_{\mathrm{ex}}\right)-1\right]^{-1}-0.16\right)}\right]
$$

where $T_{0}=h v / k=5.29$ for ${ }^{13} \mathrm{CO}$, and the excitation temperature is found based on the peak intensity, $I_{\text {peak }}$, of ${ }^{12} \mathrm{CO}$ for each pixel, using the relation

$$
T_{\mathrm{ex}}=\frac{5.53}{\ln \left[1+5.53 /\left(I_{\text {peak }}+0.82\right)\right]} .
$$

We found that the excitation temperature is $T_{\mathrm{ex}}=20 \pm 4 \mathrm{~K}$ in our map. At each pixel position and velocity, the column density can be determined according to the following equation (Wilson et al. 2009):

$$
N_{13}(x, y, v)=\left(2.5 \times 10^{14}\right) T_{\mathrm{ex}} \frac{\tau_{13}(x, y, v) d v}{1-\exp \left(-T_{0} / T_{\mathrm{ex}}\right)} .
$$

The column density summed over all velocity channels is $N_{13}(x, y)=\Sigma_{\text {vel }} N_{13}(x, y, v)$. The outflow (molecular hydrogen) mass at each position pixel is given by $M(x, y)=$ $m_{\mathrm{H}_{2}} N_{\mathrm{H}_{2}}(x, y) A$, where $m_{\mathrm{H}_{2}}$ is the mean molecular weight taking into account the abundance of helium and other trace constituents, in this case 2.72 times the mass of a hydrogen atom, and $N_{\mathrm{H}_{2}}=7 \times 10^{5} N_{13}$ (Frerking et al. 1982) is the molecular hydrogen column density. The ratio of $\left[\mathrm{H}_{2} /{ }^{13} \mathrm{CO}\right]$ is an important source of uncertainty in the calculations. Some studies found this ratio to be about 0.5 times the value we use here (e.g., Pineda et al. 2008), so the outflow masses may be less according to this factor, but here we use the value of $7 \times 10^{5}$ from Frerking et al. (1982) to allow for more straightforward comparisons with other similar studies of the same outflows that we present here. $A$ is the physical area of the pixel at the distance of the source; in our case each pixel is $2^{\prime \prime} \times 2^{\prime \prime}$, which corresponds to $5 \times 10^{31} \mathrm{~cm}^{2}$. Finally, the mass summed over the outflow area is $M=\sum_{\text {area }} M(x, y)$.

Outflow momentum $\left(P_{\text {out }}\right)$ and energy $\left(E_{\text {out }}\right)$ are found according to the following relations, respectively:

$$
\begin{gathered}
P_{\text {out }}=\Sigma_{\text {vel }} M(v)\left|v-v_{\text {cloud }}\right| \\
E_{\text {out }}=\frac{1}{2} \Sigma_{\text {vel }} M(v)\left|v-v_{\text {cloud }}\right|^{2},
\end{gathered}
$$

where $M(v)$ is the outflow lobe mass for a given velocity channel, $v$ is the velocity corresponding to that channel, and $v_{\text {cloud }}=8 \mathrm{~km} \mathrm{~s}^{-1}$ is the cloud velocity. We sum over all velocity channels with $\left|v-v_{\text {cloud }}\right|>2 \mathrm{~km} \mathrm{~s}^{-1}$. Characteristics of outflows associated with known protostellar sources and candidate outflows are itemized in Table 5, where we have made no correction for inclination of the outflow with respect to the plane of the sky.

In total, we measure $6 M_{\odot}$ of gas that we associate with outflow lobes or outflow candidates. One source of uncertainty that propagates in our mass estimate, and consequently momentum and energy, is the excitation temperature. The approach described here allows for non-constant excitation temperature throughout the region mapped, calculating excitation temperature pixel-by-pixel. As mentioned previously, we find a mean excitation temperature of $20 \mathrm{~K}$. If we assume a constant $T_{\mathrm{ex}}=20 \mathrm{~K}$, we calculate masses for each outflow that are $95 \% \pm 6 \%$ of those calculated following the pixel-by-pixel method described previously. Outflows typically have $T_{\mathrm{ex}} \sim$ $10-50 \mathrm{~K}$, and we follow several studies which suggest that the outflows in Perseus, and NGC 1333 in particular, likely have temperatures at the lower limit of this range (e.g., Knee \& Sandell 2000). Other studies of the NGC 1333 region (e.g., Hatchell et al. 2007a; Curtis et al. 2010b, and references therein) used a higher excitation temperature $T_{\mathrm{ex}}=50 \mathrm{~K}$, acknowledging $T_{\mathrm{ex}}$ up to $100 \mathrm{~K}$. Using a constant $T_{\mathrm{ex}}=$ $50 \mathrm{~K}$, we calculate outflow masses approximately twice those reported here.

We acknowledge that within the $49 \operatorname{arcmin}^{2}\left(0.23 \mathrm{pc}^{2}\right)$ area of the map, there are a few regions where we detect $\mathrm{CO}$ emission with a signal-to-noise ratio greater than 3 , which we cannot associate with any of the identified or candidate outflows presented here. It is possible that some outflows are un-identifiable due to resolution or in the case that some YSOs beyond the edges of our map are driving outflows which extend into the region which we mapped. We suspect that with improved resolution, and by mapping a larger surrounding area, we will be able to better associate and quantify the outflow emission in this region.

We calculate the total momentum and energy for identified outflows and outflow candidates to be $19 M_{\odot} \mathrm{km} \mathrm{s}^{-1}$ and $7.2 \times 10^{44} \mathrm{erg}$, respectively. Assuming an average inclination angle with respect to the line of sight $\xi=57^{\circ} .3$ (following Bontemps et al. 1996; Nakamura et al. 2011) we estimate the total outflow momentum and energy (corrected by outflow inclination angle) to be $35 M_{\odot} \mathrm{km} \mathrm{s}^{-1}$ and $2.5 \times 10^{45} \mathrm{erg}$, respectively.

\section{DISCUSSION}

\subsection{Area and Volume Occupied by Outflow Emission}

From Figure 4 we can see that $\mathrm{CO}$ outflow emission covers much of the area mapped with highest sensitivity (see also Figure 1). At first glance this seems to indicate that outflows in this region could have a major impact on the environment. In order to quantify this, we estimated the percentage of the area of the cloud in the mapped region that corresponds to outflows. We detect $\mathrm{CO}$ emission with $\left|v_{\text {out }}\right|>2 \mathrm{~km} \mathrm{~s}^{-1}$ and with a signal-to-noise ratio greater than three in $70 \%$ of the area we mapped projected on the plane of the sky. This two-dimensional diagnostic gives a sense that outflows occupy a major fraction of the cluster, and as a further diagnostic of outflows within the three-dimensional cluster, we also compare outflow volume with cloud volume according to the following method.

We estimate the volume of each outflow based on the ellipses we fit to the emission associated with identified outflows and outflow candidates described in Section 3.2 (see Table 4). We assumed that the outflow lobes in three dimensions are approximately ellipsoidal such that the depth is equal to the minor axis of the two-dimensional ellipse on the plane of the sky. Without correcting for inclination in the plane of the sky, our estimate is a lower limit to the outflow volume, the most drastic case being IRAS 4B (see Section 3.2.3) which is likely oriented along the line of sight, and therefore whose depth is likely substantially larger than its width on the plane of the sky. Further, we reiterate that unlike the calculation of area occupied by outflows for which we include all $\mathrm{CO}$ emission with $\left|v_{\text {out }}\right|>2 \mathrm{~km} \mathrm{~s}^{-1}$ that we detect in the region we mapped, in the calculation of outflow volume we measure only outflows 
(and outflow candidates) that we explicitly identify in the region and fit with ellipsoids.

We estimate the volume of the cloud within the region mapped by our CARMA observations, which we assume to be the approximate center and most active region of the cluster, to be $0.1-0.3 \mathrm{pc}^{3}$ based on the following assumptions. We assume that the depth of the cloud in this region is equal to $D=0.8-2 \mathrm{pc}$ $\left(12^{\prime}-30^{\prime}\right.$ at a distance of $235 \mathrm{pc}$ ), corresponding to the minor axis of the cloud determined from $\mathrm{C}^{18} \mathrm{O}$ observations (Ridge et al. 2003) and ${ }^{13} \mathrm{CO}$ observations (Arce et al. 2010), respectively. We approximate the volume of the cloud within the mapped region to be an ellipsoid such that $V=(4 / 3)(A)(D / 2)$, where $A=0.23 \mathrm{pc}^{2}$ is the area of our map. Including ellipsoids fit to all the identified outflows and outflow candidates (see Table 4), we calculate that in the region we mapped the volume of the cloud occupied by outflows is $\sim 2 \%-6 \%$.

This volume filling factor is much less than the area filling factor, leading us to conclude that even in the very active central region of the cluster, the majority of the volume is not occupied by outflows during the early stages of star formation that we observe here. However, this does not necessarily negate the possibility that outflows impact the surrounding large-scale cluster gas, perhaps with the aid of magnetic fields acting as an important agent that helps sustain outflow-driven turbulence (e.g., Wang et al. 2010). Since most of the sources driving outflows in the region are Class 0 , with time the outflows will occupy more of the cloud volume as they become less collimated and more wide-spread. If we assume that all outflows in our map will eventually have the volume of the Class I source SVS 13, then the 22 outflow lobes would potentially occupy up to $30 \%$ of the region's volume in the next $\sim 0.5 \mathrm{Myr}$, the lifetime for the Class I stage reported by Evans et al. (2009).

\subsection{Energy Imparted by Outflows}

IRAC images and our millimeter observations show the prevalence of outflows within this region, implying that indeed these outflows have an impact on the surrounding cloud. From the discussion in Sections 3.2 and 3.3 and the values listed in Table 5, it is apparent that the identified outflows have a range of morphologies and energetics which contribute to the total outflow activity in the region. In order to quantify the impact of this outflow activity, we need to compare the total outflow energetics with gravitational energy and turbulence within the cloud according to the method described below.

We calculate the cloud mass within the region mapped by our CARMA observations (see Figure 4 ) to be $M_{\mathrm{cl}}=140 M_{\odot}$, using the FCRAO ${ }^{13} \mathrm{CO}$ data for the same region and $T_{\mathrm{ex}}=13 \mathrm{~K}$ (Arce et al. 2010). We estimate the gravitational binding energy $\left(W=-G M_{\mathrm{cl}}^{2} / R_{\mathrm{cl}}\right)$ to be $7.2 \times 10^{45} \mathrm{erg}$ in this region. We assume a cloud radius $\left(R_{\mathrm{cl}}\right)$ of $0.24 \mathrm{pc}$, which is half the extent of the $7^{\prime}$ mapped region. The energy associated with identified outflows (with no inclination angle accounted for) equals about $10 \%$ of the gravitational energy in the region, and about one-third when using an average outflow inclination angle of $\xi=57.3$. Although outflow energy does not exceed the region's gravitational energy, the outflows that we detect will likely cause mass to escape from the cloud given that the outflows have velocities greater than the escape velocity of $v_{\mathrm{esc}}=2.2 \mathrm{~km} \mathrm{~s}^{-1}$ for the region (Arce et al. 2010). Given that up to $30 \%$ of the cloud volume will eventually be occupied by outflowing gas (see previous section), and assuming a homogeneous density for the cloud, about $30 \%$ of the mass will be in outflows with the potential to escape. Since gravitational binding energy is proportional to $M^{2}$, the gravitational energy will decrease by a factor of $(0.7)^{2} \approx 50 \%$ during the next $\sim 0.5 \mathrm{Myr}$.

We also investigate the impact outflows have on the turbulence of the cloud. Based on cloud mass $M_{\mathrm{cl}}=140 M_{\odot}$ and average ${ }^{13} \mathrm{CO}$ velocity width (FWHM) $\Delta V=2.2 \mathrm{~km} \mathrm{~s}^{-1}$ (Arce et al. 2010), we find that $E_{\text {turb }}=(3 / 16) \ln (2) M_{\text {cloud }} \Delta V_{\text {turb }}^{2}=$ $1.8 \times 10^{45} \mathrm{erg}$. This equals about one quarter of the gravitational potential energy for the same region, and is approximately 2.5 times greater than the total energy of identified outflows in the region (without correction for inclination angle). Depending on outflow inclination angle, the outflow energy may be approximately equal to turbulent energy. Indeed outflows have the potential to contribute energy comparable to the amount of turbulence we measure in the region, and therefore outflows may be an important turbulent agent.

A further diagnostic of outflows and turbulence compares outflow luminosity and turbulent dissipation rate (i.e., the power needed to maintain the turbulence in the cloud), taking into account the outflow and turbulent energies and timescales according to the following method. We begin by assuming an outflow timescale equal to the mean dynamical time of outflows in the region. Our estimate of mean dynamical time is $\sim 5 \times 10^{4} \mathrm{yr}$, calculated as $t_{\text {dyn }}=\left\langle l_{\text {out }}\right\rangle / v_{\text {char }}$, where $\left\langle l_{\text {out }}\right\rangle=$ $0.15 \mathrm{pc}$ is the mean outflow length from Table $4\left(\left\langle l_{\text {out }}\right\rangle=0.27 \mathrm{pc}\right.$, with $\xi=57.3)$, and $v_{\text {char }}=P_{\text {out }} / M_{\text {out }}=3.1 \mathrm{~km} \mathrm{~s}^{-1}$ $\left(v_{\text {char }}=5.8 \mathrm{~km} \mathrm{~s}^{-1}\right.$, with $\left.\xi=57.3\right)$.

In estimating the outflow timescale, we make admittedly simplistic assumptions, including unimpeded outflow motion along a straight path and with constant velocity. To validate this adopted timescale we also estimate lower and upper limits for the timescale. Assuming a typical jet velocity of $100 \mathrm{~km} \mathrm{~s}^{-1}$ (i.e., Arce et al. 2010) and an average outflow length of $0.15 \mathrm{pc}$ (as above), a lower limit on the age of the outflow is $1.5 \times 10^{3} \mathrm{yr}$. As an upper limit for the lifetime of most sources that drive outflows in this region, we adopt $0.5 \mathrm{Myr}$ which is the lifetime for the Class I stage reported by Evans et al. (2009). All identified outflows in our map are driven by Class 0 objects, except for SVS 13A and IRAS 2B which are likely very young Class I objects. Given the span of nearly two orders of magnitude for the lower and upper limits for the timescales stated here, we consider the mean dynamical time of $t_{\text {dyn }} \sim 5 \times 10^{4}$ yr to be a reasonable median value, acknowledging the uncertainty in this value as cautioned by Arce et al. (2010), and we use this as the outflow timescale, $\tau_{\text {out }}$, in the following estimate of outflow luminosity.

We calculate the outflow luminosity to be $L_{\text {out }}=E_{\text {out }} / \tau_{\text {out }}=$ $5 \times 10^{32} \mathrm{erg} \mathrm{s}^{-1}$ (or $L_{\text {out }}=2 \times 10^{33} \mathrm{erg} \mathrm{s}^{-1}$, with $\xi=57.3$ ). Turbulent dissipation rate is given by $L_{\text {turb }}=E_{\text {turb }} / t_{\text {diss }}$, where $t_{\text {diss }}=5.7 \times 10^{5} \mathrm{yr}$ is the energy dissipation time for NGC 1333 from Arce et al. (2010). For the region we mapped, we calculate $L_{\text {turb }}=10^{32} \mathrm{erg} \mathrm{s}^{-1}$, yielding a ratio $r_{L}=L_{\text {out }} / L_{\text {turb }} \sim 5$. As is the case for the majority of outflow regions studied by Arce et al. (2010), the ratio $r_{L}$ is greater than one, signifying that in this region the outflows have more than enough power to be an important agent for the maintenance of turbulence.

These energy diagnostics pertain to only the region we mapped, which is the central part of the cluster. Even if outflows indeed disrupt the central region, we cannot conclude that they disrupt the entire cluster, for which we would need to map a larger area. However, our results imply that the star formation activity will be seriously affected in the central part of the cluster, and if this area is disrupted and dispersed, star formation could be halted in the most active and densest region within the cloud. 


\subsection{Protostellar Sources within the Region}

Within our mapped region of $\sim 0.23 \mathrm{pc}^{2}$, we detect 9 continuum sources, and a total of 22 outflow lobes or features. The continuum sources have masses of $\sim 0.1-2 M_{\odot}$ (see Table 3 , and discussion in Section 3.1). Of these nine continuum sources, six have associated outflows. In regard to the 22 outflow lobes and features, these are driven by 12 distinct sources, of which 8 are previously identified protostellar sources, and 4 are driven by unidentified sources (i.e., outflow candidates C1, C2, C3 and C4). Within the same region are 55 YSOs and 3 starless cores (see Table 2). The YSOs include 11 Class 0 sources, 11 Class I sources, 4 flat-SED sources, 25 Class II sources, and 4 Class III sources. Of these, 31 YSOs (7 Class 0, 4 Class I, 3 flat-SED, 16 Class II, and 1 Class III) and 1 starless core reside within the highest sensitivity region (inner $\sim 6^{\prime} \times 6^{\prime}$; see Figure 1).

Considering the relatively small number of sources in our region, we do not aim to present statistically significant trends, but rather a qualitative discussion of sources and ages within the region in the context of clustered star formation. Of the 55 YSOs in the entire map, we identify outflows associated with 6 (of 11) Class 0 sources and 2 (of 11) Class I sources. We find no outflow-driving sources beyond Class I. Furthermore, we note that the two outflow-driving Class I sources that we identify are likely very young Class I sources. We also note that seven Class I sources (of the 11 considered here) and four Class 0 sources (SK 1, SK 18, IRAS 4C, IRAS 4D) lie within the outer region of our map where we have lower sensitivity, and therefore we would need to extend the mapped region to better investigate the outflow emission associated with these sources. In particular, the candidate outflow lobes $\mathrm{C} 3$ and $\mathrm{C} 4$ appear to originate near the Class I source IRAS 7 . If we consider only the inner $6^{\prime} \times 6^{\prime}$ region with best sensitivity, 2 out of 4 (or $50 \%$ ) of the Class I, and 5 out of 7 (or $70 \%$ ) of the Class 0 sources in this region drive identified outflows.

In a study of the $\mathrm{CO}$ data in the FCRAO Taurus Molecular Cloud survey, Narayanan et al. (2012) found that $75 \%$ of Class 0 sources, $30 \%$ of Class I sources and $12 \%$ of flat-SED sources known within the Taurus region drive outflows. Our detection rate within the region of NGC 1333 that we observed with best sensitivity is consistent with that of Narayanan et al. (2012), especially considering that we mapped a much smaller area (and our sample is smaller).

We propose several explanations for why we associate outflows with a greater percentage of younger rather than older objects. First, outflow morphology and collimation changes with time, with the younger outflows more collimated, and the older outflows having wider opening angles (Arce et al. 2007). While we expect that all Class 0 and Class I sources should drive outflows, it is possible that within the lifetime of a Class I source ( $~ 0.5 \mathrm{Myr}$ ), an outflow's morphology becomes diffuse enough as to become much less feasible to associate outflow emission with its driving source. Proper motions of outflow sources (e.g., Bally \& Reipurth 2001; Goodman \& Arce 2004) may also make it harder to identify older sources with their respective outflows.

Alternatively, if outflows are episodic by nature, then perhaps the episodes are more intermittent at later evolutionary stages, and the emission from outflow events has had time to propagate farther from their driving sources (and to slow down), making association of outflows more difficult for these sources. Based on the sources in the region mapped with highest sensitivity, we associate outflows with 5/7 Class 0 sources but only 2/4 Class I sources. Assuming that all Class 0 and Class I sources indeed drive outflows, and given a Class 0 lifetime of $0.17 \mathrm{Myr}$ (Enoch et al. 2009) and Class I lifetime of 0.5 Myr (Evans et al. 2009), we crudely estimate that outflows are "on" an average of about $50 \%-70 \%$ of the time during the Class 0 and I stages, or about $\sim 0.1 \mathrm{Myr}$ and $\sim 0.25 \mathrm{Myr}$, respectively. This is consistent with the results of Narayanan et al. (2012) which indicate that outflows are relatively short-lived based on the non-detection of outflows in majority of the Class I and flat-SED sources in their map.

Interestingly, one of the Class 0 sources in our map for which we do not identify an outflow (IRAS 4D) has values of $\alpha$ and $T_{\text {bol }}$ from Evans et al. (2009) which indicate that it is a very young Class 0 source, similar to SK 1, which drives an outflow with very "knotty," likely episodic emission. If in fact IRAS 4D and SK 1 are younger Class 0 sources in our map, and considering the average time in which outflows are "on" during each phase, then we suggest that the beginning of the Class 0 phase drives short bursts of outflow, followed by progressively longer-duration outflows throughout the Class 0 and Class I stages. Based on these arguments, our observations indicate that the relative ages of regions may be correlated with the fraction of Class 0 and Class I sources driving outflows, particularly more collimated outflows. We also acknowledge that the issue of associating outflows with their driving sources is exacerbated in clustered star-forming regions like NGC 1333, where emission is already confused among multiple coincident outflows.

In summary, we detect 9 continuum sources, and outflows are driven by 12 distinct sources in our map. This corresponds to 35 continuum sources and about 50 outflow-driving sources per square parsec. It is also possible that some sources outside of the mapped region are driving outflows which flow into this region, or conversely that sources near the edges of the maps drive outflows outside of the region, so this value is only approximate and will likely become more significant when a larger region is mapped. Nonetheless, these diagnostics confirm that this is a very active region of star formation. In addition, there is at least one starless core (HRF51; Hatchell et al. 2007b) in the region we mapped (with highest sensitivity), which may also form one or more protostars that will drive outflows, adding to the volume occupied by outflows as well as momentum and energy of the region over time.

\subsection{Mean Outflow Properties, Compared with Models}

Average mass, momentum, and energy of all outflow lobes and features in our map are $0.3 M_{\odot}, 0.9 M_{\odot} \mathrm{km} \mathrm{s}^{-1}$, and $3 \times 10^{43} \mathrm{erg}$, respectively. Considering just bipolar outflows, the average mass, momentum, and energy of outflows (of both lobes) are $0.7 M_{\odot}, 2 M_{\odot} \mathrm{km} \mathrm{s}^{-1}$, and $8 \times 10^{43} \mathrm{erg}$, respectively. Knee \& Sandell (2000) calculate a total of $1 M_{\odot}, 10 M_{\odot} \mathrm{km} \mathrm{s}^{-1}$, and $1 \times 10^{45}$ erg in outflows, assuming an arbitrary mean outflow inclination of $45^{\circ}$. They identify 10 outflow-exciting sources in their $\sim 63 \operatorname{arcmin}^{2}$ map, corresponding to mean outflow mass, moment, and energy of approximately $0.1 M_{\odot}, 1 M_{\odot} \mathrm{km} \mathrm{s}^{-1}$, and $1 \times 10^{43} \mathrm{erg}$, respectively. In comparison, correcting for an inclination of $45^{\circ}$, we measure mean outflow mass, moment, and energy of $0.7 M_{\odot}, 3 M_{\odot} \mathrm{km} \mathrm{s}^{-1}$, and $16 \times 10^{43} \mathrm{erg}$, respectively, larger than the values presented by Knee \& Sandell (2000). The difference likely arises from the different method used to estimate mass by Knee \& Sandell (2000). We believe that our method, which uses ${ }^{13} \mathrm{CO}$ to correct for the velocity dependent opacity of the ${ }^{12} \mathrm{CO}$ line (see Section 3.4 ) results in more reliable mass estimates (e.g., Offner et al. 2011).

Some models of outflows, and in particular outflow-driven turbulence, parameterize outflow strength assuming that momentum is proportional to stellar mass (e.g., Matzner \& McKee 
2000; Nakamura \& Li 2007). Matzner \& McKee (2000) calculate wind momentum as $p_{w}=f_{w} v_{w} m_{*}$, where the fraction $f_{w}$ of the final mass $m_{*}$ of the protostar driving the wind gives the wind mass $m_{w}$, and $v_{w}$ is wind velocity. They justify the assumption that neither $f_{w}$ nor $v_{w}$ depends on mass, based on the $\mathrm{X}$-wind model of Shu et al. (1994), and values of $f_{w}=1 / 3$ and $f_{w}=1 / 10$ correspond to the $\mathrm{X}$-wind and disk wind theories, respectively. In their simulations, Nakamura \& Li (2007) adopt a value of $f=0.5$ in their standard models, also exploring values of $f=0.25-0.75$.

For an average YSO mass of $0.5 M_{\odot}$ (Evans et al. 2009) and inclination corrected (with $\xi=57.3$ ) mean bipolar outflow momentum of $4 M_{\odot} \mathrm{km} \mathrm{s}^{-1}$, we estimate $f \sim 0.08$, assuming conservation of momentum between wind and molecular outflow (basically, entrained cloud gas) and $v_{w}=100 \mathrm{~km} \mathrm{~s}^{-1}$. Uncertainty in $f$ may be due to our choices of $v_{w}$ and excitation temperature, discussed in Section 3.4, each resulting in uncertainties in the momentum estimate of a factor of about two. We assume that all of the protostellar wind's momentum is used to drive the observed molecular outflow, which should be the case due to conservation of momentum, but perhaps the molecular outflow does not fully trace the wind's momentum. Nakamura \& $\mathrm{Li}$ (2007) use higher values of $f$ in their simulations, although they acknowledge that their value for $p_{w}$ is likely biased toward high velocities and that weaker outflows are difficult to study in detail, whereas our estimates may be biased toward lower values because of the velocity coverage of our observations, reaching only outflow velocities of $\left|v_{\text {out }}\right| \lesssim 10 \mathrm{~km} \mathrm{~s}^{-1}$, and we also do not know the correction for outflow inclination.

Of course, our observations are of one particular young region with an age of about $1 \mathrm{Myr}$ (and where outflow sources are in the Class 0 and Class I stages), and therefore it is likely that more momentum will be imparted by outflows as the sources in this region evolve. In order to quantify the effect of this, we assume that outflows drive constant momentum during the Class 0 and Class I stages, and that these stages have durations of $0.2 \mathrm{Myr}$ and 0.5 Myr, respectively (Enoch et al. 2009; Evans et al. 2009). This leads us to estimate that the outflows we observe here may last for about two to three times the current outflow driving time. Consequently, the total momentum from outflows may be up to three times the current momentum in this region, and we suggest an upper estimate of $f \sim 3 \times 0.08=0.24$.

Since we and others find average outflow momentum in NGC 1333 to be on the lower end of the outflow momentum implemented in models of turbulence injection in protostellar clusters, we suggest that lower values of $f$ are more consistent with observations. Thus, we propose that models should investigate values of $f$ in the range $\sim 0.1-0.25$, in order to understand the impact of outflows during Class $0 /$ I. Higher values of $f$, such as those with $f=0.25-0.75$ in the simulations of Nakamura \& Li (2007), do not seem to be supported based on our observations. We also suggest that simulations implement episodic outflows (discussed in the previous section), rather than instantaneous or constant injection of momentum, based on our results.

\section{SUMMARY}

We observed the protostellar region NGC 1333 using CARMA, and combining with single dish observations from FCRAO, we present mosaic maps that are sensitive on scales from $\sim 5^{\prime \prime}$ to the map size $\sim 7^{\prime}(0.006-0.5 \mathrm{pc}$ at a distance of $235 \mathrm{pc}$ ). In this paper we focus on the ${ }^{12} \mathrm{CO}$ emission which traces cool molecular outflows, and we use simultaneous observations of ${ }^{13} \mathrm{CO}$ in order to obtain reliable mass estimates. We identify a total of 22 outflow lobes and features, of which 18 comprise 9 identified bipolar outflows, while 4 outflow features have no identified bipolar counterpart, likely because these outflows extend beyond the edges of our map. We detect 9 continuum sources in our map, of which 6 are responsible for driving a total of 14 outflow lobes that we identify, with one of these sources driving perpendicular outflows.

The spatial $\left(\sim 5^{\prime \prime}\right)$ and spectral $\left(0.3 \mathrm{~km} \mathrm{~s}^{-1}\right)$ resolution of our observations facilitate our detailed description of outflow morphologies in the region, including identifying outflow-driving sources where possible, as well as evidence for interaction between the outflows in this dense clustered region. We detect $\mathrm{CO}$ emission in $70 \%$ of the $49 \operatorname{arcmin}^{2}\left(0.23 \mathrm{pc}^{2}\right)$ area of the map, and we estimate an outflow volume filling factor of $2 \%-6 \%$. We calculate a total of $6 M_{\odot}$ of gas associated with identified outflows and outflow candidates, compared with a cloud mass of $140 M_{\odot}$ corresponding to the region we mapped.

We calculate kinematics of the individual identified outflows within the region, as well as total outflow momentum and energy, which are $19 M_{\odot} \mathrm{km} \mathrm{s}^{-1}$ and $7.2 \times 10^{44} \mathrm{erg}$, respectively $\left(35 M_{\odot} \mathrm{km} \mathrm{s}^{-1}\right.$ and $2.5 \times 10^{45} \mathrm{erg}$, respectively, assuming an average inclination angle with respect to the line of sight of 57.3 for all outflows). Comparing with gravitational binding energy and turbulent energy for the same region, we find that outflow energy is comparable to turbulent energy, and about onethird of the gravitational potential energy, assuming an average outflow inclination angle of $\xi=57.3$. We also calculate the ratio of outflow to turbulent luminosity, which is greater than one. These diagnostics lead us to suggest that outflows act as an important agent for the maintenance of turbulence in this region. If enough mass is ejected by outflows in excess of the cloud escape velocity, the gravitational binding energy of the cloud could diminish to the point that outflow energetics substantially disrupt the region of the cluster which we mapped, where most stars are forming.

We also investigate characteristics of protostars and outflows in the region mapped, where about $30 \%$ of the YSOs within all of NGC 1333 reside. We identify outflows associated with 5 out of 7 Class 0 and 2 out of 4 Class I sources within the region mapped with the highest sensitivity. We suggest that while we expect that all Class 0 and Class I sources should drive outflows, with our observations we are not able to associate outflows with all of these sources due to (1) difficulty identifying outflow features as they become older and less-collimated, and/or (2) more sporadic outflow episodicity at later stages. The challenge of identifying outflow features is only augmented in a clustered region like the prototypical NGC 1333, but this is a pertinent issue considering most stars form in embedded cluster environments.

Finally, we make several suggestions of parameters that are important for simulations of clustered star-forming regions. In the region we mapped, the average mass, momentum, and energy of outflow lobes and features are $0.3 M_{\odot}, 0.9 M_{\odot} \mathrm{km} \mathrm{s}^{-1}$, and $3 \times 10^{43} \mathrm{erg}$, respectively (with no inclination correction). Our results support a lower value of $f_{w}$, which parameterizes outflow strength, than has been implemented in previous simulations. In other words, about $10 \%-25 \%$ of the final mass of a protostar comprises the protostellar wind, which in turn drives momentum in the cluster environment. Models should take these parameters into account when investigating the impact of outflows during the first $\sim 0.5 \mathrm{Myr}$ of protostellar evolution. Our results pertain specifically to the central, most active region of clustered star formation, and we suggest that the dynamics of this 
region are critical in understanding the broader impact, and particularly the dispersal, by outflows within the larger cluster environment.

We thank an anonymous referee for suggestions that strengthened the quality of the paper. A.L.P. thanks J. Carpenter for help with data reduction, and M. Pound for advice on combining interferometer and single dish maps. This material is based upon work supported under a National Science Foundation Graduate Research Fellowship, and support from the U.S. Student Program of Fulbright Chile. This project was funded by the NSF under grant AST-0845619 to H.G.A. D.M. gratefully acknowledges support from CONICYT project BASAL PFB-06. Support for CARMA construction was derived from the Gordon and Betty Moore Foundation, the Kenneth T. and Eileen L. Norris Foundation, the James S. McDonnell Foundation, the Associates of the California Institute of Technology, the University of Chicago, the states of California, Illinois, and Maryland, and the NSF. Ongoing CARMA development and operations are supported by the NSF under a cooperative agreement, and by the CARMA partner universities. The National Radio Astronomy Observatory is a facility of the National Science Foundation operated under cooperative agreement by Associated Universities, Inc.

\section{Facilities: CARMA, FCRAO}

\section{REFERENCES}

Arce, H. G., Borkin, M. A., Goodman, A. A., Pineda, J. E., \& Beaumont, C. N 2011, ApJ, 742, 105

Arce, H. G., Borkin, M. A., Goodman, A. A., Pineda, J. E., \& Halle, M. W. 2010, ApJ, 715, 1170

Arce, H. G., \& Goodman, A. A. 2001, ApJ, 554, 132

Arce, H. G., \& Goodman, A. A. 2002a, ApJ, 575, 928

Arce, H. G., \& Goodman, A. A. 2002b, ApJ, 575, 911

Arce, H. G., \& Sargent, A. I. 2006, ApJ, 646, 1070

Arce, H. G., Shepherd, D., Gueth, F., et al. 2007, in Protostars and Planets V, ed. B. Reipurth, D. Jewitt, \& K. Keil (Tucson, AZ: Univ. Arizona Press), 245

Aspin, C., Sandell, G., \& Russell, A. P. G. 1994, A\&AS, 106, 165

Bachiller, R., Codella, C., Colomer, F., Liechti, S., \& Walmsley, C. M. 1998, A\&A, 335, 266

Bachiller, R., Gueth, F., Guilloteau, S., Tafalla, M., \& Dutrey, A. 2000, A\&A, 362, L33

Bally, J., Devine, D., \& Reipurth, B. 1996, ApJL, 473, L49

Bally, J., \& Reipurth, B. 2001, ApJ, 546, 299

Bally, J., Reipurth, B., Lada, C. J., \& Billawala, Y. 1999, AJ, 117, 410

Banerjee, R., Klessen, R. S., \& Fendt, C. 2007, ApJ, 668, 1028

Benedettini, M., Molinari, S., Testi, L., \& Noriega-Crespo, A. 2004, MNRAS, 347,295

Blake, G. A., Sandell, G., van Dishoeck, E. F., et al. 1995, ApJ, 441, 689

Bontemps, S., Andre, P., Terebey, S., \& Cabrit, S. 1996, A\&A, 311, 858

Carroll, J. J., Frank, A., Blackman, E. G., Cunningham, A. J., \& Quillen, A. C. 2009, ApJ, 695, 1376

Černis, K. 1990, Ap\&SS, 166, 315

Chandler, C. J., \& Richer, J. S. 2000, ApJ, 530, 851

Chen, X., Arce, H. G., Zhang, Q., et al. 2013, ApJ, 768, 110

Chen, X., Bourke, T. L., Launhardt, R., \& Henning, T. 2008, ApJL, 686, L107

Chen, X., Launhardt, R., \& Henning, T. 2009, ApJ, 691, 1729

Chini, R., Reipurth, B., Sievers, A., et al. 1997, A\&A, 325, 542

Choi, M. 2001, ApJ, 553, 219

Choi, M. 2005, ApJ, 630, 976

Cunningham, A. J., Frank, A., Carroll, J., Blackman, E. G., \& Quillen, A. C. 2009, ApJ, 692, 816

Curtis, E. I., Richer, J. S., \& Buckle, J. V. 2010a, MNRAS, 401, 455

Curtis, E. I., Richer, J. S., Swift, J. J., \& Williams, J. P. 2010b, MNRAS, 408, 1516

Davis, C. J., Cervantes, B., Nisini, B., et al. 2011, A\&A, 528, A3

Davis, C. J., Scholz, P., Lucas, P., Smith, M. D., \& Adamson, A. 2008, MNRAS, 387,954 de Zeeuw, P. T., Hoogerwerf, R., de Bruijne, J. H. J., Brown, A. G. A., \& Blaauw, A. 1999, AJ, 117,354

Engargiola, G., \& Plambeck, R. L. 1999, in Proc. 3rd Cologne-Zermatt Symp. The Physics and Chemistry of the Interstellar Medium, ed. V. Ossenkopf, J. Stutzki, \& G. Winnewisser (Waabs: GCA-Verlag), 291

Enoch, M. L., Evans, N. J., II, Sargent, A. I., \& Glenn, J. 2009, ApJ, 692, 973

Enoch, M. L., Young, K. E., Glenn, J., et al. 2006, ApJ, 638, 293

Evans, N. J., II, Dunham, M. M., Jørgensen, J. K., et al. 2009, ApJS, 181, 321

Foster, P. N., \& Boss, A. P. 1996, ApJ, 468, 784

Frerking, M. A., Langer, W. D., \& Wilson, R. W. 1982, ApJ, 262, 590

Girart, J. M., Crutcher, R. M., \& Rao, R. 1999, ApJL, 525, L109

Goodman, A. A., \& Arce, H. G. 2004, ApJ, 608, 831

Gueth, F., \& Guilloteau, S. 1999, A\&A, 343, 571

Gutermuth, R. A., Myers, P. C., Megeath, S. T., et al. 2008, ApJ, 674, 336

Hartmann, L., Ballesteros-Paredes, J., \& Bergin, E. A. 2001, ApJ, 562, 852

Haschick, A. D., Moran, J. M., Rodriguez, L. F., et al. 1980, ApJ, 237, 26

Hatchell, J., \& Dunham, M. M. 2009, A\&A, 502, 139

Hatchell, J., Fuller, G. A., \& Richer, J. S. 2007a, A\&A, 472, 187

Hatchell, J., Fuller, G. A., Richer, J. S., Harries, T. J., \& Ladd, E. F. 2007b, A\&A, 468, 1009

Hatchell, J., Richer, J. S., Fuller, G. A., et al. 2005, A\&A, 440, 151

Herbig, G. H., \& Jones, B. F. 1983, AJ, 88, 1040

Hirota, T., Bushimata, T., Choi, Y. K., et al. 2008, PASJ, 60, 37

Jennings, R. E., Cameron, D. H. M., Cudlip, W., \& Hirst, C. J. 1987, MNRAS, 226,461

Jørgensen, J. K., Harvey, P. M., Evans, N. J., II, et al. 2006, ApJ, 645, 1246

Knee, L. B. G., \& Sandell, G. 2000, A\&A, 361, 671

Lada, C. J., Alves, J., \& Lada, E. A. 1996, AJ, 111, 1964

Lada, C. J., \& Lada, E. A. 2003, ARA\&A, 41, 57

Ladd, E. F., Adams, F. C., Fuller, G. A., et al. 1991, ApJ, 382, 555

Langer, W. D., \& Penzias, A. A. 1993, ApJ, 408, 539

Launhardt, R. 2004, in IAU Symp. 221, Star Formation at High Angular Resolution, ed. M. G. Burton, R. Jayawardhana, \& T. L. Bourke (Cambridge: Cambridge Univ. Press), 213

Lay, O. P., Carlstrom, J. E., \& Hills, R. E. 1995, ApJL, 452, L73

Lee, C.-F., \& Ho, P. T. P. 2005, ApJ, 624, 841

Lee, C.-F., Mundy, L. G., Stone, J. M., \& Ostriker, E. C. 2002, ApJ, 576, 294

Lefloch, B., Castets, A., Cernicharo, J., Langer, W. D., \& Zylka, R. 1998, A\&A, 334, 269

Liseau, R., Sandell, G., \& Knee, L. B. G. 1988, A\&A, 192, 153

Looney, L. W., Mundy, L. G., \& Welch, W. J. 2000, ApJ, 529, 477

Lynds, B. T. 1962, ApJS, 7, 1

Maret, S., Bergin, E. A., Neufeld, D. A., et al. 2009, ApJ, 698, 1244

Matzner, C. D. 2007, ApJ, 659, 1394

Matzner, C. D., \& McKee, C. F. 2000, ApJ, 545, 364

Moriarty-Schieven, G. H., Snell, R. L., Strom, S. E., et al. 1987, ApJ, 319,742

Nakamura, F., \& Li, Z.-Y. 2007, ApJ, 662, 395

Nakamura, F., Sugitani, K., Shimajiri, Y., et al. 2011, ApJ, 737, 56

Narayanan, G., Snell, R., \& Bemis, A. 2012, MNRAS, 425, 2641

Neufeld, D. A., \& Yuan, Y. 2008, ApJ, 678, 974

Offner, S. S. R., Lee, E. J., Goodman, A. A., \& Arce, H. 2011, ApJ, 743, 91

Padoan, P., Juvela, M., Kritsuk, A., \& Norman, M. L. 2009, ApJL, 707, L153

Pineda, J. E., Caselli, P., \& Goodman, A. A. 2008, ApJ, 679, 481

Plambeck, R. L., \& Engargiola, G. 2000, in ASP Conf. Ser. 217, Imaging at Radio through Submillimeter Wavelengths, ed. J. G. Mangum \& S. J. E. Radford (San Francisco, CA: ASP), 354

Raga, A. C., Noriega-Crespo, A., Carey, S. J., \& Arce, H. G. 2013, AJ, 145, 28

Reipurth, B., Yu, K. C., Rodríguez, L. F., Heathcote, S., \& Bally, J. 1999, A\&A, 352, L83

Ridge, N. A., Di Francesco, J., Kirk, H., et al. 2006, AJ, 131, 2921

Ridge, N. A., Wilson, T. L., Megeath, S. T., Allen, L. E., \& Myers, P. C. 2003, AJ, 126,286

Rodríguez, L. F., Anglada, G., \& Curiel, S. 1997, ApJL, 480, L125

Rodríguez, L. F., Anglada, G., \& Curiel, S. 1999, ApJS, 125, 427

Sandell, G., Aspin, C., Duncan, W. D., Russell, A. P. G., \& Robson, E. I. 1991, ApJL, 376, L17

Sandell, G., \& Knee, L. B. G. 2001, ApJL, 546, L49

Sandell, G., Knee, L. B. G., Aspin, C., Robson, I. E., \& Russell, A. P. G. 1994, A\&A, 285, L1

Sault, R. J., Teuben, P. J., \& Wright, M. C. H. 1995, in ASP Conf. Ser. 77, Astronomical Data Analysis Software and Systems IV, ed. R. A. Shaw, H E. Payne, \& J. J. E. Hayes (San Francisco, CA: ASP), 433

Schnee, S., Enoch, M., Johnstone, D., et al. 2010, ApJ, 718, 306

Schnee, S., Sadavoy, S., Di Francesco, J., Johnstone, D., \& Wei, L. 2012, ApJ, 755, 178 
Shu, F., Najita, J., Ostriker, E., et al. 1994, ApJ, 429, 781

Shu, F. H., Adams, F. C., \& Lizano, S. 1987, ARA\&A, 25, 23

Snell, R. L., \& Bally, J. 1986, ApJ, 303, 683

Stanimirovic, S. 2002, in ASP Conf. Ser. 278, Single-dish Radio Astronomy: Techniques and Applications, ed. S. Stanimirovic, D. Altschuler, P. Goldsmith, \& C. Salter (San Francisco, CA: ASP), 375

Strom, S. E., Vrba, F. J., \& Strom, K. M. 1976, AJ, 81, 314

Terquem, C., Eislöffel, J., Papaloizou, J. C. B., \& Nelson, R. P. 1999, ApJL, 512, L131
Walawender, J., Bally, J., Francesco, J. D., Jørgensen, J., \& Getman, K. 2008 in The Handbook of Star Forming Regions, Vol. I: The Northern Sky, ed. B. Reipurth (ASP Monograph Publ., Vol. 4; San Francisco, CA: ASP), 346

Wang, P., Li, Z.-Y., Abel, T., \& Nakamura, F. 2010, ApJ, 709, 27

Wilson, T. L., Rohlfs, K., \& Hüttemeister, S. (ed.) 2009, Tools of Radio Astronomy (Berlin: Springer)

Yıldız, U. A., Kristensen, L. E., van Dishoeck, E. F., et al. 2012, A\&A, 542, A86

Yu, K. C., Billawala, Y., \& Bally, J. 1999, AJ, 118, 2940 